\title{
Continuous bubble streams for controlling marine biofouling on static artificial structures
}

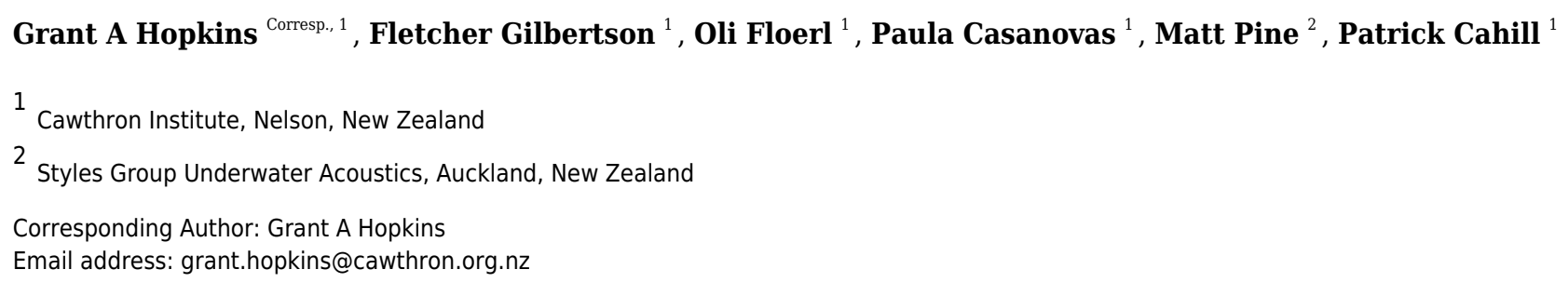

Biofouling accumulation is not proactively managed on most marine static artificial structures (SAS) due to the lack of effective options presently available. We describe a series of laboratory and field trials that examine the efficacy of continuous bubble streams in maintaining SAS free of macroscopic biofouling and demonstrate that this treatment approach is effective on surface types commonly used in the marine environment. At least two mechanisms were shown to be at play: the disruption of settlement created by the bubble stream, and the scouring of recently settled larvae through shear stress. Field trials conducted over a one-year period identified fouling on diffusers as a major issue to longterm treatment applications. Field measurements suggest that noise associated with surface mounted air blowers and sub-surface diffusers will be highly localised and of low environmental risk. Future studies should aim to develop and test systems at an operational scale. 
1 Title: Continuous bubble streams for controlling marine biofouling on static artificial structures

2

3 Grant A. Hopkins*1, Fletcher R. Gilbertson ${ }^{1}$, Oliver Floerl ${ }^{1}$, Paula Casanovas ${ }^{1}$, Matthew K.

$4 \quad$ Pine $^{2}$, Patrick L. Cahill ${ }^{1}$

5

$6{ }^{1}$ Cawthron Institute, Private Bag 2, Nelson 7042, New Zealand

$7 \quad 2$ Styles Group Underwater Acoustics

8 * Corresponding author: grant.hopkins@cawthron.org.nz

\section{ABSTRACT}

11 Biofouling accumulation is not proactively managed on most marine static artificial structures

12 (SAS) due to the lack of effective options presently available. We describe a series of laboratory

13 and field trials that examine the efficacy of continuous bubble streams in maintaining SAS free

14 of macroscopic biofouling and demonstrate that this treatment approach is effective on surface

15 types commonly used in the marine environment. At least two mechanisms were shown to be at

16 play: the disruption of settlement created by the bubble stream, and the scouring of recently

17 settled larvae through shear stress. Field trials conducted over a one-year period identified

18 fouling on diffusers as a major issue to long-term treatment applications. Field measurements

19 suggest that noise associated with surface-mounted air blowers and sub-surface diffusers will be

20 highly localised and of low environmental risk. Future studies should aim to develop and test

21 systems at an operational scale. 
23 Keywords: Air bubbles, marinas, static structures, settlement, treatment 


\section{INTRODUCTION}

Harbours throughout the world have been heavily modified and contain a vast surface area of static artificial structures (SAS) associated with ports, marinas and other facilities (Dugan et al. 2011; Firth et al. 2016). These surfaces are quickly colonised by biological fouling (biofouling) communities that are often dominated by non-indigenous species (Glasby et al. 2007, Airoldi et al. 2015). For some structures, fouling can be costly to manage and lead to a range of undesirable outcomes, including biocorrosion (e.g., on oil rigs and wind farms), increased loading and hydrodynamic drag (e.g., for pontoons and warps), and crop or stock losses in aquaculture (Bannister et al. 2019). For structures like wharf piles and marina pontoons, the financial incentives to prevent or remove fouling are less evident and fouling is often left unmanaged.

The establishment and spread of marine pests within coastal systems is closely monitored in countries like New Zealand, Australia and the United States, and ports and marinas are typically the focal points for systematic surveys. However, SAS associated with ports and marinas are rarely actively maintained or treated to prevent the establishment and proliferation of biofouling, except in the case of pest eradication and population control efforts (Forrest \& Hopkins 2013). Biofouling accumulations on SAS are generally extensive and result in highly elevated recruitment rates to resident or visiting vessels or other submerged structures (Floerl \& Inglis 2003, 2005). Reducing biofouling on SAS will reduce the need for periodic and expensive biofouling-related maintenance of infrastructure. This will reduce the likelihood of marine pest establishment in vector hubs (i.e., act as a barrier to new incursions), and interrupt subsequent spread by reducing resident vessel fouling rates (i.e., through reduced propagule supply). These indirect benefits of managing biofouling on SAS associated with ports and marinas, as well as other artificial environments such as aquaculture farms, are in addition to direct improvements in 
48 structural integrity and longevity by minimising biofouling-related hydrodynamic drag and

49 microbially-induced corrosion.

50 The arsenal of tools to manage SAS biofouling is limited, with mechanical approaches (e.g.,

51 water blasters, scrapers, mechanic brushes) the most widely available. The present suite of

52 antifouling paints available on the market are generally not amenable to use on SAS, with the

53 three main classes of soluble-matrix coatings, self-polishing copolymers, and silicone- or

54 fluoropolymer-fouling release coatings all requiring water flows above threshold values to

55 remain effective. In addition, periodic removal of SAS for antifouling coating renewal would in

56 many cases be unfeasible. Therefore, the most common approaches to fouling management on

57 SAS is to do nothing and accept the consequences or to rely on physical removal methods.

58 Several studies have highlighted the potential effectiveness of bubble streams (or curtains) in controlling biofouling accumulation on artificial surfaces in the marine environment. Scardino et al. (2009) tested the efficacy of bubble streams over acrylic and fouling release panels held in a V-shaped frame to replicate the submerged portion of a vessel hull, followed by a field trial on a hull section of a commercial vessel. Significant differences were observed between treated and untreated panels; however, some macrofouling accumulation (e.g., hydroids) occurred despite treatment. Similarly, the hull section of the vessel being treated was largely devoid of macrofouling (covering only $10 \%$ of the experimental surface area) compared to the adjacent non-treated areas of the hull ( $88 \%$ macrofouling cover), although a slime layer still developed. Bullard et al. (2010) investigated the efficacy of continuous bubble exposure applied to PVC and concrete panels deployed over relatively short periods ( 1 and 4-week deployments) at three sites with different seabed communities. Like Scardino et al. (2009), rates of macrofouling development were significantly lower on panels treated with bubbles ( $4 \%$ of that on controls 
71 after 4 weeks). Lowen et al. (2016) tested the effects of bubble streams and suspended particles

72 on the settlement and survivorship of early life-stages of Ciona intestinalis. Larval settlement

73 was effective at flows $>10 \mathrm{~L} \mathrm{~min}^{-1}$ (with $0 \%$ recruitment at $20 \mathrm{~L} \mathrm{~min}^{-1}$ ), but juveniles that had

74 settled for 21 days were resilient to treatment. The authors concluded that continuous treatment

75 would be required to prevent Ciona establishment on structures.

76 In contrast to these earlier investigations, which involved exposing surfaces to a 'cloud' of

77 bubbles, Menesses et al. (2017) undertook laboratory and field investigations into the minimum

78 shear stress required for a continuous single bubble stream. This study concluded that shear

79 stress forces of around $0.01 \mathrm{~Pa}$ are required to prevent biofouling accumulation.

Despite the early promise shown by bubble stream approaches to biofouling management described above, there has been no real-world uptake by biofouling managers or technology developers. This lack of uptake is likely because key knowledge gaps remain. Firstly, the mode or mechanism of treatment is unclear. Studies to date have primarily focused on shear stresses created by bubble streams dislodging adhered fouling organisms (e.g., Menesses et al. 2017). We hypothesise that a continuous stream of bubbles also has the potential to disrupt the settlement process. Here we define disruption as either: (1) creating a physical barrier, by the movement of many bubbles or the formation of a large bubble on the experimental surfaces, or (2) the instantaneous removal of larvae from the surface before they are able to settle. Secondly, there is a lack of guidance around crucial operational requirements for effective treatment, such as bubble stream flow rates, frequency and duration of application. Artificial surfaces in the marine

91 environment include a diversity of surface types (e.g., concrete, polyethylene, wood) and

92 orientations, and it is unclear how bubble stream performance could be impacted by this

93 diversity. Finally, although bubble streams likely present a lower environmental risk than 
94 traditional antifouling coatings (Bullard et al. 2010), there is a growing body of evidence

95 identifying detrimental impacts of underwater noise pollution on marine mammals and fish (see

96 Jones 2019; Slabbekoorn et al. 2010). Quantifying noise levels generated by bubble-based

97 treatment systems is therefore necessary prior to large-scale implementation.

98 The present study describes a series of laboratory and field trials that collectively aimed to

99 address the knowledge gaps identified above. We investigate whether treatment efficacy could

100 be enhanced through changing the surface type of SAS and/or angle of application. We also

101 investigate underwater noise emissions generated from our field test system, as this would

102 arguably be the key environmental risk factor associated with widespread use. Collectively our

103 experiments explore whether bubble streams could be an effective, environmentally benign

104 treatment method for SAS and stationary vessels with the intent to facilitate the uptake of

105 bubble-based biofouling prevention technologies. 
106

107

108

109

110

111

112

113

114

115

116

117

118

119

120

121

122

123

124

125

126

127

128

\section{MATERIALS AND METHODS}

LABORATORY TRIALS

The purpose of the laboratory experiments described below was to explore mechanisms of action, as well as inform decisions around treatment parameters applied in subsequent field trials (e.g., flow rate, angle of application, surface type).

\section{Testing system and surface types}

Laboratory trials were undertaken in a temperature-controlled room $\left(18 \pm 1^{\circ} \mathrm{C}\right)$. The experimental apparatus comprised a 478-L tank (L x W x H: 1115 x 715 x 600 mm) with two Hyotube $^{\mathrm{TM}}$ Series 9 fine bubble diffusers (model number 9-200EP-KPH-4; pore size = $1 \mathrm{~mm}$; Ecologix Technology) placed on the bottom of the tank (Fig. 1). The diffusers were powered by a blower (K04 MS MOR 1.1kW; FPZ Blower Technology). An aluminium frame, fixed ca. 400 $\mathrm{mm}$ directly above the diffusers, held the experimental panels in place (horizontally) during treatment. Flow rates from the diffusers were manipulated by venting excess air from the blower to the atmosphere via a ball valve. Air flow rates were estimated by inverting a $1000-\mathrm{mL}$ measuring cylinder filled with water above the diffusers at the depth of the experimental panels, and the time taken to displace $500 \mathrm{~mL}$ of water recorded. Prior to each trial, flows were measured and adjusted to achieve either high $\left(2.6 \mathrm{~L} \mathrm{~h}^{-1} \mathrm{~cm}^{-2}\right)$, medium $\left(1.7 \mathrm{~L} \mathrm{~h}^{-1} \mathrm{~cm}^{-2}\right)$, or low $\left(0.9 \mathrm{~L} \mathrm{~h}^{-1} \mathrm{~cm}^{-2}\right)$ flow rates. Estimated shear stresses created by the bubble streams within the testing system under these different flow scenarios are provided in the Online Supplementary Material (S1). Our system also included a second control tank that was identical to the treatment tank except it did not have air diffusers or air pumped into the water. Two panel types were used in the experiments: plain black acrylic panels with a matt texture finish (ACR), and acrylic

PeerJ reviewing PDF | (2020:11:55476:2:0:NEW 28 Mar 2021) 
129 panels professionally coated with the fouling release coating Intersleek 1100SR (FR-IS1100,

130 International Paint). Panel dimensions were 200 x 150 mm (W x L).

\section{Model organisms}

133 Two model organisms were used to explore bubble stream modes of action: the Pacific transparent sea squirt Ciona savignyi and the Pacific oyster Crassostrea gigas. Both species are non-indigenous to New Zealand and are considered nuisance fouling organisms. In our experiments, Ciona larvae were ready to settle onto experimental surfaces within $24 \mathrm{~h}$ of hatching, whereas Crassostrea larvae were reared for $17 \mathrm{~d}$ prior to settling. Detailed spawning, rearing and larvae settling procedures are provided in the Online Supplementary Material (S2).

Mechanisms of action: scouring

141 The ability of bubble streams to 'scour' settled larvae was investigated by pre-settling Ciona (an estimated density of 0.5 individuals $\mathrm{cm}^{-2}$ based on counts from control panels) and Crassostrea larvae $\left(0.3\right.$ individuals $\left.\mathrm{cm}^{-2}\right)$ onto experimental panels and quantifying removal efficacy for variations of surface type (fixed, 2 levels: ACR and FR-IS1100), settlement time prior to treatment (2 levels, fixed: 3 and $120 \mathrm{~h}$ ), and flow rates (4 levels, fixed: 0, 0.9, 1.7 and $2.5 \mathrm{~L} \mathrm{~h}^{-1}$ $\mathrm{cm}^{-2}$ ). These factors were assessed in a fully-crossed experimental design with $\mathrm{n}=5$ experimental panels for each treatment combination (Fig. 1). Larvae settlement times, flow rates and the treatment duration parameters used in these experiments were selected based on extensive pilot work (not presented in this paper) using the same treatment system and model 150 species. 
151 The ACR and FR-IS1100 panels were treated simultaneously across the four flow rates (order 152 randomised) and two settlement times ( 3 and $120 \mathrm{~h}$ ), with bubble stream treatment lasting 10 153 minutes. Panels settled with Ciona larvae for $3 \mathrm{~h}$ were placed in a 300-L recirculating holding 154 tank for 5 days following treatment (i.e., until the $120 \mathrm{~h}$ settlement panels had also been treated).

155 Ciona larvae are lecithotrophic and therefore did not requiring feeding within this timeframe. By

156 contrast, the $3 \mathrm{~h}$ Crassostrea panels were placed in a $300 \mathrm{~L}$ non-recirculating aerated tank

157 following treatment, and were fed approximately $5 \mathrm{~L}$ of Isochrysis galbana (approx. 10 million

158 cells $\mathrm{mL}^{-1}$ ) daily for 5 days, with a $50 \%$ water change undertaken after 2 days. At the completion 159 of the $120 \mathrm{~h}$ treatments, the entire surface of all panels (including the $3-\mathrm{h}$ settlement panels in 160 holding tanks) was inspected using a binocular microscope (10 x magnification) and the number 161 of settled larvae recorded.

\section{Mechanisms of action: settlement disruption}

164 To test our hypothesis that bubble streams disrupt settlement, we compared larval settlement 165 success on blank ACR and FR-IS1100 panels $(n=6$ per treatment combination, Fig. 1) placed in 166 two tanks (with and without bubble streams) filled with Ciona (ca. 50 larvae $\mathrm{L}^{-1}$ ) and 167 Crassostrea (ca. 10,500 larvae $\mathrm{L}^{-1}$ ) larvae. Each species was tested separately. Panels in the treatment tank were subjected to a medium intensity of bubbling $\left(1.7 \mathrm{~L} \mathrm{~h}^{-1} \mathrm{~cm}^{-2}\right)$ for a period of

$16924 \mathrm{~h}$. During the Crassostrea trial, 20 L of Isochrysis galbana (approx. 10 million cells $\mathrm{mL}^{-1}$ )

170 was added to each of the tanks. After $24 \mathrm{~h}$, panels were inspected under a dissecting microscope 171 (x10 magnification) for the presence of settled larvae. 
FIELD TRIALS

174 Vertical surfaces

175 Twenty-four $300 \times 400 \mathrm{~mm}$ panels were fixed vertically to four stainless steel frames suspended

$1761 \mathrm{~m}$ beneath a floating dock in Port Nelson (S $\left.41^{\circ} 15^{\prime}, \mathrm{E} 173^{\circ} 16^{\prime}\right)$. Twelve panels were

177 constructed from concrete (CONC; a non-disclosed commercial marina pontoon formulation);

178 the other twelve panels were constructed from acrylic professionally coated with a fouling

179 release coating (FR-IS1 100). Two of the experimental frames (each holding $\mathrm{n}=3 \mathrm{CONC}, \mathrm{n}=3$

180 FR-IS1100) were continuously exposed to a bubble curtain treatment for 13 weeks. To achieve

181 this, a $1.1 \mathrm{kw}$ blower delivered $76 \mathrm{~m}^{3}$ air $\mathrm{h}^{-1}$ to two pairs (one pair per treated frame) of Hyotube

182 fine bubble diffusers (see laboratory trials for blower and diffuser details). The diffusers were

183 mounted $100 \mathrm{~mm}$ below the experimental panels (compared with $400 \mathrm{~mm}$ in the laboratory

184 trials) to compensate for the strong tidal currents present in the field. Two treatment control

185 frames, each holding three CONC and three FR-IS1100 panels, received no bubble treatment

186 (Fig. 2).

187 Panels were visually inspected in situ after 1, 4, 6, and 9 weeks by a scientific diver familiar with

188 biofouling assessments, and a categorical level of fouling (LoF; based on Floerl et al. 2005) was

189 assigned. At the completion of the experiment (week 13), each panel was photographed (Canon

190 PowerShot G16, 12.1 megapixels), the last visual LoF was assigned, and biofouling removed

191 using a plastic scraper for biomass assessments.

192 Photoquadrat images of the experimental panels were analysed using the random dot method

193 (Meese \& Tomich 1992) in Coral Point Count software (CPCe v4.1, Kohler \& Gill 2006), with

19450 stratified random points overlaid on each image. Sessile taxa $>1 \mathrm{~mm}$ were identified to major

195 taxonomic groups (barnacles, ascidians, bryozoans, tubeworms, oysters, mussels, hydroids, 
196 sponges, biofilm/bare space), and in the case of ascidians, further categorised based on their

197 morphology (solitary vs colonial). A margin of $2 \mathrm{~cm}$ around each experimental unit was

198 excluded from analyses to avoid edge effects. Fouling biomass was measured following air

199 drying to a constant weight $\left(60^{\circ} \mathrm{C}, 72 \mathrm{~h}\right)$.

\section{Horizontal and angled surfaces}

202 Between October 2018 and September 2019, the efficacy of continuous bubble streams was evaluated on horizontal and angled surfaces from a series of trials at the Devonport Naval Base, Auckland. A purpose-built raft was constructed using aluminium framing and polyethylene floats (1400 mm long, $700 \mathrm{~mm}$ diameter) for buoyancy (Fig. 3). The raft was fitted with 8 diffusers connected to a blower (K05 MS MOR 1.5kW; FPZ Blower Technology) via a series of hoses (40 mm internal diameter) and 2:1 hose connectors. Each diffuser hose line was fitted with a ball valve so that flow rates could be independently adjusted. For all trials, flow rates were set to correspond to the high flow used in the laboratory experiments (i.e., $2.6 \mathrm{~L} \mathrm{~h}^{-1} \mathrm{~cm}^{-2}$ ). The blower was also fitted with a variable frequency drive (Invertek Optidrive, Model ODE-2-12150$1 \mathrm{~KB} 1 \mathrm{X})$ so that delivery of total air flow to the diffusers could also be adjusted.

A single diffuser was suspended $300 \mathrm{~mm}$ directly below each of 8 panels $(500 \mathrm{~mm} \times 750 \mathrm{~mm})$ representing four surface types: concrete (CONC), black polyethylene (POLY), acrylic coated with International Paint FR coatings Intersleek 1100SR (FR-IS1100) and Intersleek 1000 (FRIS 1000). For each of the four surface types, one panel was fixed at $0^{\circ}$ (flat) and $22^{\circ}$ (angled). Due to space limitations, the first three trials had one replicate for each surface type:orientation combination (i.e., replication was achieved by undertaking three trials sequentially). Positions on

218 the raft were randomly assigned for each trial. Following these trials, the accumulation of 
219 biofouling on POLY and FR-IS1000 panels with and without bubble treatment was assessed

220 (Fig. 3). Panels were fixed in a flat orientation and were deployed for three months, with $\mathrm{n}=2$

221 panels for both treatments and controls.

222 After each deployment period the experimental raft was lifted from the water, panels were

223 removed and photographed (Canon PowerShot SX280 HS, 12 megapixels), and new panels

224 fitted. Photoquadrat images of the experimental panels were analysed using the random dot

225 method in Coral Point Count software, with 200 stratified random points overlaid on each image.

226 Sessile taxa $>1 \mathrm{~mm}$ were identified to major taxonomic groups and their percentage cover

227 estimated. A margin of $2 \mathrm{~cm}$ around each experimental unit was excluded from analyses to avoid

228 edge effects. Significant fouling development on the diffusers resulted in 'shadows' of treatment,

229 and fouling could be observed directly above areas of the diffuser where this occurred. These

230 patches of fouling were excluded from analyses $(\min =0 \%$ of data points per panel, $\max =$

$23147.0 \%$, average $=9.7 \%$ ). All panel images are provided in the Online Supplementary Material

$232(\mathrm{~S} 5)$.

233

234 Underwater noise

235 The use of a surface mounted blower and the release and subsequent collapse of microbubbles

236 creates underwater noise. To gain insights into potential issues (e.g., impacts on marine

237 mammals), underwater noise measurements were obtained from a bubble stream system

238 deployed at Nelson Marina. The diffusers, powered by a $1.1 \mathrm{kw}$ blower, were suspended $1 \mathrm{~m}$

239 below the water surface from a marina pontoon. Conditions within the marina during the trial

240 were calm with an incoming tide of approximately 5 to $7 \mathrm{~cm} \mathrm{~s}^{-1}$. Sound traps (ST 300 STD units)

241 were deployed on temporary moorings at 1-m and 3-m water depths adjacent to the diffusers, 3- 
$242 \mathrm{~m}$ water depth $16 \mathrm{~m}$ from the diffusers, and at 3-m water depth $32 \mathrm{~m}$ from the diffusers. Acoustic

243 recordings of the system were collected as follows: 10 minutes without the blower turned on

244 (ambient); 7 minutes with the blower running, but not connected to the diffusers; 7 minutes with

245 the blower running, and connected to the diffusers with maximum air flow; 7 minutes with the

246 blower running, and connected to the diffusers with medium air flow (comparable to flow rates

247 used during field trials); then 10 minutes without the blower turned on (ambient).

248 During each trial, a cabled hydrophone (Cetacean Research Technology CR1) was placed near to

249 the diffusers to check for any extraneous noise contamination, such as an approaching vessel.

250 Disrupted trials were discontinued and repeated after confirming the contaminating noise source

251 was no longer detectable. Acoustic recordings were processed in MATLAB, where the power

252 spectrum for each trial was calculated and plotted.

253

254 Data analyses

255 Laboratory trials

256 Generalized linear models (GLM, Dobson 1990) were used to test how flow rate, settlement

257 period and surface type affected the efficiency of the bubble treatment. For the scouring and

258 disruption trials, we modeled individual counts of Ciona and Crassostrea using a GLM with

259 negative binomial error distribution, because the ratio between the residual deviance and the

260 degrees of freedom showed that Poisson models were over-dispersed. For each trial, a separate

261 model was constructed for each species. 
263 Field trials

264 Vertical surfaces

265 We performed an ordinal mixed effect model (Agresti 2002) to test how surface type (CONC or FR-IS1100) and the bubble treatment affected the level of fouling (LoF) over the experimental

267

268

269

270

271

272

273

274

275

276

277

278

279

280

281

282

283

period. An interaction term was included to test for a combined effect of the two experimental

factors. Because repeated observations were made over a 13-week period, week number was

added as a random effect in the models. A beta regression was used to test how surface type and bubble treatment affected the percent cover of macrofouling at the completion of the experimental period. A GLM (with Gamma error distribution) was used to explore factors affecting fouling biomass accumulation (assessed as dry weight). An interaction term was included in the last two models.

\section{Horizontal and angled surfaces}

For the first three 'rounds' of the field trials, GLMs were used to test how surface type and orientation angle affected the efficiency of the bubble treatment in preventing biofouling accumulation on experimental panels (measured as biofouling percent cover). An interaction term was included in the models to test for a combined effect of the experimental factors.

Because each round was undertaken during a different time period, experimental round was added as a fixed effect in the GLMs to account for seasonality. We separately modeled the percent cover of bare space, biofilm and macrofouling using a GLM with a binomial error distribution. Lastly, when the effect of bubble treatment was tested against control panels (two 
284 surface types, $0^{\circ}$ orientation), the effects size was so large that the data were simply plotted and 285 described.

286 All statistical analyses were performed within the ' $R$ ' statistical and programming environment 287 (R Development Core Team 2019). 


\section{RESULTS}

290 LABORATORY TRIALS

291 Mechanisms of action: scouring

292 Ciona settlement onto the FR-IS1100 panels proved unreliable as larvae 'slid off' the panel when 293 tipped into the vertical position after the settling period, so these data were removed from the 294 analyses. Settlement onto the acrylic panels was successful. Larval removal was significant for 295 both the 3 and $120 \mathrm{~h}$ settlement periods when exposed to high bubble flow rates, with reduction 296 of $>90 \%$ of settled larvae relative to the controls (Fig. 4, Table S3.1, p-value $<0.001$ ). Medium and low flow rates were not different to the treatment controls, and we did not find an interaction of settlement period and flow rate.

In contrast to the Ciona trials, there were no differences between the number of Crassostrea individuals remaining on the acrylic surface after treatment at any flow rate, regardless of settlement time. However, all bubble flow rates reduced the number of Crassostrea individuals (79-97\% relative to the treatment controls) when individuals were settled for 120 hours on the FR-IS1100 surface (Online Supplementary Material, Table S3.1, p-value < 0.05).

\section{Mechanisms of action: settlement disruption}

Panels exposed to continuous bubble streams resulted in nil settlement by Ciona over the $24 \mathrm{~h}$ treatment period, whereas settlement on control panels ranged from 16 to 115 and 64 to 851 larvae per panel for FR-IS1100 and ACR, respectively (Fig. 5). The continuous bubble stream also resulted in a significant reduction ( $\mathrm{p}$-value $<0.001)$ in Crassostrea settlement (average of 
311 (range $=163-774$ larvae per panel). While statistically significant (Online Supplementary

312 Material, Table S3.2), the treatment effect was not 100\% effective for Crassostrea.

FIELD TRIALS

315 Vertical surfaces

316 Biofouling accumulation on control (non-bubbled) CONC panels occurred rapidly, with

317 considerable fouling $(\mathrm{LoF}=4)$ reached after 1 week, and extensive fouling $(\mathrm{LoF}=5)$ evident

318 after 1 month (Fig. 6C). During this same period, control FR-IS1100 panels were initially

319 colonized by numerous small calcareous tubeworms $(\mathrm{LoF}=3)$. After 3 months, other fouling

320 taxa were also observed on the plates, including hydroids, tubeworms, bryozoans and ascidians.

321 CONC panels receiving bubble treatment were first colonized by a slime layer with small

322 numbers (1-3 per panel) of juvenile oysters. Over time, the number and size of oysters increased

323 because once settled they were impervious to treatment, and other species (e.g., small mussels

324 and hydroids) were found in low densities ( $\mathrm{LoF}=2)$. FR-IS1 100 panels receiving the bubble

325 treatment had a slime layer present after 4 weeks $(\mathrm{LoF}=1)$ and remained devoid of

326 macrofouling for the remainder of the trial. There was a significant interaction between surface

327 type and treatment (all p-values $<0.001$, Table S3.3), with higher LoF scores observed on the

328 concrete control panels.

329 Fouling biomass reflected the LoF scoring (Fig. 6A), with highest levels recorded on the

330 untreated CONC panels $(15.6 \mathrm{~g} \pm 1.6 \mathrm{~g}$, mean $\pm 1 \mathrm{SE})$, compared to $0.2 \mathrm{~g} \pm 0 \mathrm{~g}$ on the treated FR-

331 IS1100 panels. Oyster growth on the treated CONC panels contributed to an average biomass of

$3323.0 \mathrm{~g}(\mathrm{SE}=0.2)$, while the untreated FR-IS1100 panels averaged only $0.7 \mathrm{~g}$ per panel $(\mathrm{SE}=0.1)$.

333 Macrofouling percent cover closely matched the results for biomass (Fig. 6B). Biofouling dry 
334 weights and percent cover of macrofouling at the end of the experiment differed significantly

335 between treatment and control panels, and a significant interaction between the surface type and

336 treatment was observed (significant p-values $<0.001$, Table S3.3).

Horizontal and angled surfaces

339 Continuous bubble streams applied to the four surface types (CONC, POLY, FR-IS1100 and FR-

340 IS 1000) fixed in two orientations $\left(0^{\circ}\right.$ and $\left.22^{\circ}\right)$ kept macrofouling coverage low (average $=2 \%$,

$341 \mathrm{SE}=0.01 \%$ ), but biofilms developed on all surfaces (ranging between 27-100\%; Fig. 7).

342 Macrofouling taxa settling onto panels was restricted to barnacles and Pacific oysters. The

343 temporal component was more important in determining the percent cover of bare space, biofilm

344 and macrofouling than surface type or plate angle; round 3 was significantly different than

345 rounds 1 and 2 for bare space, biofilm and macrofouling percent cover, and round 2 was

346 significantly different than round 1 for bare space and biofilm (all p-values $<0.05$, Table S3.4).

347 Biofilm cover differed significantly between some surface types and angles, with highest cover

348 observed on concrete surfaces fixed at $0^{\circ}$ (Fig. 7).

349 When the performance of bubble streams was evaluated against controls, marked differences

350 were observed (Fig. 8). Bubble treatment prevented the accumulation of macrofouling on FR-

351 IS1000 and POLY panels, except on areas where the flow of bubble streams was disturbed by

352 fouling on the diffusers of the experimental raft (excluded from the analyses). Bubble streams

353 were also observed to reduce biofilm cover on FR-IS1000 panels. By contrast, the control panels

354 for both surface types were colonized by a range of taxa, including barnacles, Pacific oysters,

355 colonial and solitary ascidians, hydroids and filamentous algae (Fig. 9). 


\section{Underwater noise}

358 At $0.5 \mathrm{~m}$ distance from the diffusers, bubble curtain noise had a bandwidth of approximately

$3591190 \mathrm{~Hz}$, spanning $10 \mathrm{~Hz}$ (minimum hydrophone sensitivity) to $1200 \mathrm{~Hz}$ (ranging between 81

360 and $111 \mathrm{~dB}$ re $1 \mu \mathrm{Pa}^{2} \mathrm{~Hz}^{-1}$ ) at maximum flow (Figure S4.1). When flow was reduced to medium,

361 the bandwidth dropped to approximately $835 \mathrm{~Hz}$ (spanning $15 \mathrm{~Hz}$ to $850 \mathrm{~Hz}$ ), as did the spectral

362 levels (to between 86 and $108 \mathrm{~dB}$ re $1 \mu \mathrm{Pa}^{2} \mathrm{~Hz}^{-1}$ ). The blower at the surface was generally non-

363 detectable over the ambient underwater soundscape. At $16 \mathrm{~m}$ from the diffusers, underwater

364 noise was partially attenuated (spectral levels peaked at approximately $86 \mathrm{~dB}$ re $1 \mu \mathrm{Pa}^{2} \mathrm{~Hz}^{-1}$ ),

365 with a detectable frequency range between approximately 150 and $500 \mathrm{~Hz}$. At $32 \mathrm{~m}$ from the

366 diffusers, noise from the diffusers had attenuated out (i.e., no noise from the diffusers was

367 detected over the ambient soundscape). 


\section{DISCUSSION}

369 Building on the prior proof-of-concept research undertaken by Scardino et al. (2009), Bullard et

370 al. (2010), Lowen et al. (2016), and Menesses et al. (2017), we have addressed key knowledge

371 gaps around operational implementation of bubble streams/curtains to control biofouling

372 accumulation on SAS. Our laboratory trials demonstrated that scouring of recently settled

373 biofouling organisms was possible using bubble streams, including oyster larvae that had settled

3745 days prior on a fouling release coating. We also demonstrated that larval settlement disruption

375 was possible at flow rates that were insufficient to scour larvae from surfaces, supporting a new

376 hypothesis for dual mechanisms of action of bubble streams. Field trials supported these

377 findings, with panels remaining largely fouling-free after 2-3 months of deployment. These

378 results are encouraging and indicate bubble streams are a potentially cost-effective, non-biocide-

379 based long-term treatment for minimising biofouling on static submerged infrastructure.

MODES OF ACTION AND FACTORS AFFECTING EFFICACY

382

383

384

385

386

387

388

389

390
The application of bubble streams over artificial surfaces limits biofouling development via at

least two modes of action: scouring of settled larvae due to shear stress, and settlement disruption due to physical disturbance. For scouring, we found that surface type, flow rate and biofouling species present were all important determinants of treatment efficacy. Direct comparisons between FR-IS1100 and ACR panels were not possible for Ciona due to inconsistent (generally low) settlement on the fouling release panels. However, treatment efficacy was significantly higher on FR-IS1100 panels compared with ACR when bubble streams were applied to panels seeded with Crassostrea. Differences in efficacy due to bubble flow rates were more evident in the ACR panels for both species, indicating that thresholds for larvae removal (from shear stress) 
391 was higher for the ACR panels with higher surface tension. Of the two species, Crassostrea

392 proved the most resistant to treatment.

393 Very few Crassostrea and no Ciona larvae recruited to virgin FR-IS1100 and ACR panels

394 subjected to continuous bubble streams applied at the medium flow, while relatively high

395 recruitment levels were observed on the control panels. Although not conclusive, these findings

396 indicate that settlement disruption could be sufficient at bubble flow rates lower than that

397 required to remove larvae that have settled for $3 \mathrm{~h}$ or greater. This supports the findings of

398 Lowen et al. (2016), who found that flow rates capable of preventing 3-day old Ciona larvae

399 from settling were insufficient to reliably remove 21-day old Ciona from petri dishes.

400 Underwater video footage of bubble streams interacting with the test panels revealed another

401 potential mechanism that could be exploited to prevent fouling accumulation on surfaces fixed in

402 a flat orientation. As bubbles came into contact flat panels, they would join to form large flat

403 bubbles that were variable in size (ranging from 2-3 $\mathrm{mm}$ up to ca. $50 \mathrm{~mm}$ in diameter and

404 several millimetres thick, Fig. 10). These trapped bubbles present a physical barrier to larval

405 settlement. This phenomenon occurred under low, medium and high flow rates, and was evident

406 on both the acrylic and fouling release panels. The formation and retention of large flat bubbles

407 on the underside of flat structures could potentially be achieved through surface design and by

408 delivering a mixture of small (providing settlement disruption and scouring mechanisms) and

409 large bubbles (creating larger, persistent air pockets). For example, marina pontoons could be

410 designed to trap air bubbles on the horizontal bottom surfaces, and essentially form a large air

411 bubble to repel biofouling settlement.

412 For both the scouring and settlement disruption trials, the FR-IS1100 coating was found to be

413 particularly amenable to treatment by bubble streams. For surfaces coated with fouling release 
414 paints, intermittent treatment (e.g., every 5 days) could be effective. If combined with the 415 exposure to intermittent bubble streams, the use of fouling release coatings may be feasible for a

416 broad range of vessel operational profiles (including vessels with extended lay-up periods), as

417 biofouling removal would not solely be reliant on drag forces while vessels are underway. While 418 application to concrete or polyethylene marina pontoons is technically feasible, consideration 419 would need to be given to initial application costs, likelihood of damage from vessels and 420 floating debris in these environments, and the longevity of coatings (see Hu et al. 2020).

421 Surfaces constructed from acrylic, polyethylene and concrete would likely require continuous 422 treatment to maximise physical disruption of larval settlement, along with high flow rates to 423 scour any settled larvae. Further, our observations of suboptimal performance against oyster 424 larvae in the laboratory and field trials suggest that regions prone to calcareous fouling may 425 require substantially higher bubble delivery rates than those applied in the present study to afford 426 long-term protection. As observed in our field trials, once established, oysters can form treatment 427 shadows as they change benthic boundary conditions on the surface, resulting in the establishment of taxa that would have otherwise been dislodged by the bubble streams. This has

429 parallels with the encrusting bryozoan Watersipora subtorquata, which can colonise vessel hulls 430 due to its tolerance to biocides and is then in turn colonised by less biocide-tolerant taxa (Floerl 431 et al. 2004). For bubble stream treatment approaches to be effective, near $100 \%$ efficacy against 432 settlement is needed, otherwise periodic interventions (i.e., fouling removal by other means) 433 would be required.

Due to the economic imperative to keep vessels free from marine growth (Schultz et al. 2011), studies have attempted to understand the forces required to dislodge biofouling while en route 436 and during periodic maintenance (Crisp et al. 1985; Swain \& Schultz 1996; Callow \& Callow 
437 2002; Finlay et al. 2002). This body of research has demonstrated that the adhesion strength of

438 early microorganisms is weak and can be dislodged by a shear stress of around $1 \mathrm{~Pa}$.

439 Macrofouling larvae settle with an initial adhesion strength of around 0.1 MPa (Yule \& Walker

440 1984), and following metamorphosis, adhesion strength can increase greatly; e.g., around $1 \mathrm{MPa}$

441 for barnacles (Crisp et al. 1985; Swain \& Schultz 1996). It is unsurprising then that vessels (or

442 surfaces) that are cleaned/groomed more frequently have been found to require lower shear

443 stresses to remove biofouling because the biofouling is likely to be less advanced/well adhered

444 (Tribou \& Swain 2010, 2015).

445 Menesses et al. (2017) examined the wall stresses required to keep panels free of fouling when

446 exposed to a single bubble stream and found that levels greater than $0.01 \mathrm{~Pa}(10 \mathrm{mPa})$ were

447 required, much less than that required to remove established fouling species. In the present study,

448 air flow to the diffusers was manipulated to achieve a range of shear stresses acting on panels

449 during the laboratory trials $(176 \pm 71 \mathrm{mPa}$ to $415 \pm 97 \mathrm{mPa}$ for low and high flow rates,

450 respectively, over panels held at $22^{\circ}$ ); these values are within the critical shear stress band

451 required to remove larvae (Koehl et al. 2013). It is therefore surprising that higher levels of

452 treatment efficacy were not observed when bubble streams were applied to pre-settled Ciona and

453 Crassostrea larvae in our laboratory trials.

454 In our study we were only able to estimate shear stress on plates held at an angle to the bubble

455 stream, as perpendicularly orientated plates created a stagnation point at the plate surface where

456 bubbles accumulate (Fig. 10) and make it impossible to calculate local velocity profiles using

457 low resolution cameras. Therefore, it is possible that, due to the horizontal positioning of the

458 panels and bubble interactions with panel coatings during trials, the shear stresses created at

459 these flows were less than that required for dislodgement to occur. Alternatively, it is also 
460 possible that larvae were within the benthic boundary layer and therefore not subjected to the

461 turbulent shear forces created at the boundary layer and free stream interface (Koehl \& Hadfield

462 2004; Massel 1999). As bubble stream flow rates increase, the height of the benthic boundary

463 layer would be expected to decrease (Cantwell 1981; Crimaldi et al. 2002), possibly explaining

464 why efficacy at the high flow rates was notably higher.

465 The influence of surface angle relative to the bubble stream delivery was not evident in our naval 466 base field trials. In theory, shear stress should have been higher on the angled $\left(22^{\circ}\right)$ compared to 467 the flat $\left(0^{\circ}\right)$ panels (Munson et al. 2013). It is likely that a lack of relationship between angle and 468 increased treatment efficacy was masked by the high flows applied to the panels, i.e., shear 469 forces generated over the flat panels were sufficient to remove (or disrupt) settling larvae.

CONSIDERATIONS FOR APPLICATION IN REAL-WORLD SETTINGS

472 The uptake of novel approaches to manage biofouling accumulation on SAS will require low473 maintenance, robust, fit-for-purpose and cost-effective systems that can be applied to a broad 474 range of structure types. Given the significant cost associated with the production and installation 475 of marinas and other marine infrastructure, there is a need to consider bubble delivery designs for 476 not only new builds, but also for retrofitting to existing structures.

\section{Bubble stream applications at greater water depths}

479 Further studies are needed if bubble stream approaches are considered for managing biofouling 480 on deep structures (e.g., oil rigs, wind turbines). With increasing water depths, air dissolution rates would increase (Woolf \& Thorpe 1991), and as bubbles rose to the surface, their volume 
482 would increase (basically doubling every atmosphere/10 m). Given that the relationships

483 described in this manuscript relate to bubble sizes of around $1 \mathrm{~mm}$ diameter generated in $<1.5 \mathrm{~m}$

484 water depth, the efficacy of this approach at depth remains untested. Further work could include

485 examining the fate and efficacy of bubbles released at depth, as well as overcoming some of the

486 logistical challenges that could arise (e.g., bubble generation and system maintenance at depth).

\section{Infrastructure, maintenance and running costs}

The trials described in this paper used off-the-shelf components for generating and delivering bubble streams to experimental panels. Blower and diffusers costs for the continuous bubble treatment totalled around US\$5000 (not including hardware for the raft) and treated approximately $3 \mathrm{~m}^{2}$. During the final trial period (ca. 4 months), an estimated $4300 \mathrm{~kW}$ h of electricity was used, equating to approx. US\$230 of electricity usage per square metre (based on New Zealand power rates). For a 500-berth marina, with an estimated submerged surface area of submerged pontoons approximating 10,500 $\mathrm{m}^{2}$ (for simplicity, assuming $10 \mathrm{~m}$ finger lengths $1 \mathrm{~m}$ in width, $10 \mathrm{~m}$ distances between fingers, 2-m walkways down the middle, and all structures having a $0.5 \mathrm{~m}$ draft), this would result in over US\$2.4 million in power costs per annum. If the experimental approach used in the present study was applied at an operational scale, electricity costs alone would be cost-prohibitive and existing alternatives (e.g., periodic fouling removal and capture by commercial divers) would be substantially cheaper. While more efficient systems could almost certainly be developed to run a much larger number of diffusers, and alternative

502 sources of power could be considered (e.g., solar, wind, wave, and currents), capital and 503 operational costs represent major challenges for uptake of this approach. 
504 Keeping the diffuser free of fouling and sediment build-up was a challenge for both field

505

506

507

508

509

510

511

512

513

514

515

516

517

518

519

520

521

522

523

524

525

526

experiments, and ongoing maintenance was required to ensure that the diffusers worked

efficiently (Fig. 11). When left unchecked during the first field experiment on vertical panels, fouling growth on the experimental frames interfered with bubble delivery, resulting in fouling accumulation on the bottom third of the experimental panels. Similar treatment 'shadows' were also observed in the centre of many of the panels deployed horizontally. Any systems deployed in marine environments will also have to withstand periodic storms, tidal currents, collision with debris and vessels, and the corrosive nature of seawater. Ideally, treatment systems will be designed so that failure at single points does not result in complete failure.

While challenges associated with diffuser fouling are significant, they could be addressed.

Recent advances in antifouling technologies, such as copper cold spray (Vucko et al. 2012), could be applied to the diffusers, which will comprise a much smaller total surface area than the structures being treated. Systems could also be programmed to periodically dislodge sediment build-up by 'blasting' a higher velocity of bubbles through the delivery tubes, as is done in waste-water treatment oxidation ponds (Rosso et al. 2008). Our laboratory trials also suggest that bubble delivery may not need to be continuous and could be timed so there is less disruption to marina users.

\section{Underwater noise}

Acoustic analyses revealed that the noise emissions were predominately low frequency (below $1200 \mathrm{~Hz}$ ) and highly localised, propagating at levels above the ambient soundscape within 10s of metres rather than 100 s of metres. Since the noise levels in this study are the raw levels, the distance within which a marine mammal or fish may detect the noise over the ambient

Peer] reviewing PDF | (2020:11:55476:2:0:NEW 28 Mar 2021) 
527 soundscape decreases further. However, it is important to note that this study involved the use of

528 only two bubble diffusers ( $1 \mathrm{~m}$ length each). If the concept is to be applied at a large scale

529 involving large numbers of diffusers (e.g., treating an entire marina), consideration would also

530 need to be given to cumulative noise effects (see Pine et al. 2014).

531 The potential effect of treatment noise on humans also warrants consideration. For example,

532 many marinas have rules controlling the noise from vessel engines, as well as devices such as

533 radios and televisions. Such rules are typically in place to reduce the amount of disturbance to

534 marina users and any nearby residents and businesses. Health effects arising from short and long-

535 term exposure to noise are also possible. Noise created by bubbles collapsing at the surface is

536 unlikely to pose a disturbance or health risk. However, noise created by pumps or blowers

537 generating the bubble streams could be an issue, and mitigation measures (e.g., the use of sound-

538 proofing materials, placement away from live-aboard vessels or residential areas) may be

539 necessary.

540

\section{CONCLUSIONS}

542 Bubble streams have the potential to effectively limit biofouling development on SAS over

543 extended periods of time. The development of cost-effective bubble delivery systems that

544 perform under a large range of environmental conditions and that require low maintenance will

545 be a formidable challenge. Future studies should aim to develop and test systems at an

546 operational scale using approaches that can be retrofitted to existing infrastructure as most

547 marine SAS have a service life of many decades. We expect an increased focus on the

548 integration of biofouling management systems into the future designs of SAS if efficacy can be

549 established at an operational scale. The development of bubble stream systems for installation 
550 below vessel berths would enable the use of non-biocidal fouling release coatings on slower

551 vessels that currently need to predominantly rely on biocidal coatings. 


\section{ACKNOWLEDGEMENTS}

553 The authors thank Sarah Strong (New Zealand Defence Force), the Dive team at the Devonport

554 Naval Base, Don McKenzie (Northland Regional Council), Samantha Happy (Auckland

555 Council), Kathy Walls (Biosecurity New Zealand), David Lamont (Bellingham Marine), David

556 Hart, Brett Wallace and Aaron Lines (Akzo Nobel, International Paint), Dave Duncan (Port

557 Nelson) and Bruce Lines (Diving Services New Zealand Limited) for logistical support and

558 funding for aspects of this project.

559

560 FUNDING STATEMENT

561 The publication of this work was supported by Cawthron's Internal Investment Fund and New

562 Zealand's Ministry of Business, Innovation and Employment funding (CAWX1904 - A toolbox 563 to underpin and enable tomorrow's marine biosecurity system).

\section{DISCLOSURE STATEMENT}

566 The authors declare that they have no competing interests. 


\section{REFERENCES}

570 Agresti A. 2002. Categorical Data Analysis. Second edition. Wiley. 372 p.

571 Airoldi L, Turon X, Perkol-Finkel S, Rius M. 2015. Corridors for aliens but not for natives:

572 effects of marine urban sprawl at a regional scale. Diversity and Distributions 21:755-

573 768. doi:10.1111/ddi.12301

574 Bannister J, Sievers M, Bush F, Bloecher N. 2019. Biofouling in marine aquaculture: a review of

575 recent research and developments. Biofouling 35:631-648.

$576 \quad$ doi:10.1080/08927014.2019.1640214

577 Bullard SG, Shumway SE, Davis CV. 2010. The use of aeration as a simple and environmentally $578 \quad$ sound means to prevent biofouling. Biofouling 26:587-593.

$579 \quad$ doi:10.1080/08927014.2010.496038

580 Callow ME, Callow JA. 2002. Marine biofouling: a sticky problem. Biologist. 49:1-5.

581 Cantwell BJ. 1981. Organized motion in turbulent flow. Annual Review of Fluid Mechanics

582 13:457-515. doi:10.1146/annurev.fl.13.010181.002325

583 Crimaldi JP, Thompson JK, Rosman JH, Lowe RJ, Koseff JR. 2002. Hydrodynamics of larval 584 settlement: the influence of turbulent stress events at potential recruitment sites.

585 Limnology and Oceanography 47:1137-1151. doi:10.4319/1o.2002.47.4.1137

586 Crisp D, Walker G, Young G, Yule A. 1985. Adhesion and substrate choice in mussels and 587 barnacles. Journal of Colloid and Interface Science 104:40-50. doi:10.1016/00219797(85)90007-4 
589 Dobson AJ. 1990. An introduction to generalized linear models. London: Chapman and Hall. $590 \quad 301 \mathrm{p}$.

591

592

593

594

595

596

597

598

599

600

601

602

603

604

605

606

607

608

Dugan JE, Airoldi L, Chapman MG, Walker SJ, Schlacher T. 2011. Pages 17-41 In: Wolanski E, McLusky D, eds. Estuarine and coastal structures: environmental effects, a focus on shore and nearshore structures. Treatise on Estuarine and Coastal Science. Academic Press. doi:10.1016/B978-0-12-374711-2.00802-0

Finlay JA, Callow ME, Schultz MP, Swain GW, Callow JA. 2002. Adhesion strength of settled spores of the green alga Enteromorpha. Biofouling 18:251-256. doi:10.1080/08927010290029010

Firth LB, Knights AM, Bridger D, Evans AJ, Mieszkowska N, Moore PJ, O’Connor NE, Sheehan EV, Thompson RC, Hawkins SJ. 2016. Ocean sprawl: challenges and opportunities for biodiversity management in a changing world. Oceanography and Marine Biology 54:193-269. doi:10.1201/9781315368597-9

Floerl O, Inglis GJ. 2003. Boat harbour design can exacerbate hull fouling. Austral Ecology 28:116-127. doi:10.1046/j.1442-9993.2003.01254.x

Floerl O, Inglis GJ. 2005. Starting the invasion pathway: the interaction between source populations and human transport vectors. Biological Invasions 7:589-606. doi:10.1007/s 10530-004-0952-8

Floerl O, Pool T, Inglis GJ. 2004. Positive interactions between nonindigenous species facilitate transport by human vectors. Ecological Applications 14:1724-1736. doi:10.1890/03-5399 
609 Floerl O, Inglis GJ, Hayden BJ. 2005. A risk-based predictive tool to prevent accidental

610 introductions of nonindigenous marine species. Environmental Management 35:765-778.

611 doi:10.1007/s00267-004-0193-8

612 Forrest BM and Hopkins GA. 2013. Population control to mitigate the spread of marine pests:

613

614

615

616

617

618

619

620

621

622

623

624

625

626

627

628

629 insights from management of the Asian kelp Undaria pinnatifida and colonial ascidian Didemnum vexillum. Management of Biological Invasions 4:317-326. doi:10.3391/mbi.2013.4.4.06

Glasby TM, Connell SD, Holloway MG, Hewitt CL. 2007. Nonindigenous biota on artificial structures: could habitat creation facilitate biological invasions? Marine Biology 151:887-895. doi:10.1007/s00227-006-0552-5

Hu P, Xie Q, Ma C, Zhang G. 2020. Silicone-based fouling-release coatings for marine antifouling. Langmuir 36:2170-2183. doi:10.1021/acs.langmuir.9b03926

Jones N. 2019. Ocean uproar: saving marine life from a barrage of noise. Nature 568:158-161. doi:10.1038/d41586-019-01098-6

Koehl MAR, Crimaldi JP, Dombroski DE. 2013. Wind chop and ship wakes determine hydrodynamic stresses on larvae settling on different microhabitats in fouling communities. Marine Ecology Progress Series 479:47-62. doi:10.3354/meps1019310.3354/meps 10193

Koehl MAR, Hadfield MG. 2004. Soluble settlement cue in slowly moving water within coral reefs induces larval adhesion to surfaces. Journal of Marine Systems 49:75-88. doi:10.1016/j.jmarsys.2003.06.003 
630 Kohler KE, Gill SM. 2006. Coral Point Count with Excel extensions (CPCe): a Visual Basic

631

632

633

634

635

636

637

638

639

640

641

642

643

644

645

646

647

648

649 program for the determination of coral and substrate coverage using random point count methodology. Computers \& Geosciences 32:1259-1269. doi:10.1016/j.cageo.2005.11.009

Lowen JB, Deibel D, McKenzie CH, Couturier C, DiBacco C. 2016. Tolerance of early lifestages in Ciona intestinalis to bubble streams and suspended particles. Management of Biological Invasions 7:157-165. doi:10.3391/mbi.2016.7.2.03

Massel SR. 1999. Fluid mechanics for marine ecologists. Springer, New York; Berlin. 566 p.

Meese RJ, Tomich PA. 1992. Dots on rocks: a comparison of percentage cover methods. Journal of Experimental Marine Biology \& Ecology 165:59-73. doi:10.1016/00220981(92)90289-M

Menesses M, Belden J, Dickenson N, Bird J. 2017. Measuring a critical stress for continuous prevention of marine biofouling accumulation with aeration. Biofouling 33:703-711. doi:10.1080/08927014.2017.1359574

Munson BR, Okiishi TH, Huebsch WW, Rothmayer AP. 2013. Fundamentals of fluid mechanics, 7th edn, John Wiley \& Sons, Inc, Hoboken, NJ.

Pine MK, Jeffs AG, Radford CA. 2014. The cumulative effect on sound levels from multiple underwater anthropogenic sound sources in shallow coastal waters. Journal of Applied Ecology 51:23-30. doi:10.1111/1365-2664.12196

R Development Core Team. 2019. R: A Language and Environment for Statistical Computing. Vienna, Austria: R Foundation for Statistical Computing. 
650 Rosso D, Larson LE, Stenstrom MK. 2008. Aeration of large-scale municipal wastewater 651 treatment plants: state of the art. Water Science \& Technology 57:973-978.

652 doi:10.2166/wst.2008.218

653 Scardino AJ, Fletcher LE, Lewis JA. 2009. Fouling control using air bubble curtains: protection 654 for stationary vessels. Journal of Marine Engineering and Technology A13: 3-10. 655 doi:10.1080/20464177.2009.11020214

656 Schultz MP, Bendick JA, Holm ER, Hertel WM. 2011. Economic impact of biofouling on a 657 naval surface ship. Biofouling 27:87-98. doi:10.1080/08927014.2010.542809

658

659

660

661

662

663

664

665

666

667

668

669

Slabbekoorn H, Bouton N, van Opzeeland I, Coers A, ten Cate C, Popper AN. 2010. A noisy spring: the impact of globally rising underwater sound levels on fish. Trends in Ecology and Evolution 25:419-427. doi:10.1016/j.tree.2010.04.005

Swain GW, Schultz MP. 1996. The testing and evaluation of non-toxic antifouling coatings. Biofouling 10:187-197. doi:10.1080/08927019609386279

Tribou M, Swain G. 2010. The use of proactive in-water grooming to improve the performance of ship hull antifouling coatings. Biofouling 26:47-56. doi:10.1080/08927010903290973

Tribou M, Swain G. 2015. Grooming using rotating brushes as a proactive method to control ship hull fouling. Biofouling 31:309-319. doi:10.1080/08927014.2015.1041021

Vucko MJ, King PC, Poole AJ, Carl C, Jahedi MZ, de Nys R. 2012. Cold spray metal embedment: an innovative antifouling technology. Biofouling 28:239-248. doi:10.1080/08927014.2012.670849 
670 Woolf DK, Thorpe SA. 1991. Bubbles and the air-sea exchange of gases in near-saturation 671 conditions. Journal of Marine Research 49:435-66. doi:10.1357/002224091784995765

672 Yule AB, Walker G. 1984. The temporary adhesion of barnacle cyprids: effects of some 673 differing surface characteristics. Journal of the Marine Biological Association of the $674 \quad$ United Kingdom 64:679-686. doi:10.1017/S0025315400030101 


\section{Figure 1}

Schematic of the treatment system used in both the scouring and settlement disruption trials.

The treatment tank contained two diffusers (side-by-side, $200 \mathrm{~mm}$ apart) that were powered by a $1.1 \mathrm{kw}$ blower. Aluminium racks were used to hold experimental panels approximately $400 \mathrm{~mm}$ above the diffusers. The control tank used in the disruption experiments was configured the same as the treatment tank, minus the diffusers and associated pipework. Experimental details for the scouring and disruption trials are shown. Note that Ciona larvae could not be reliably settled onto the fouling release panels (Intersleek 1100SR, FR-IS1100) so were excluded from the analyses. $A C R=$ acrylic panel $(200 \times 150 \mathrm{~mm})$. 
Mechanism of action: scouring

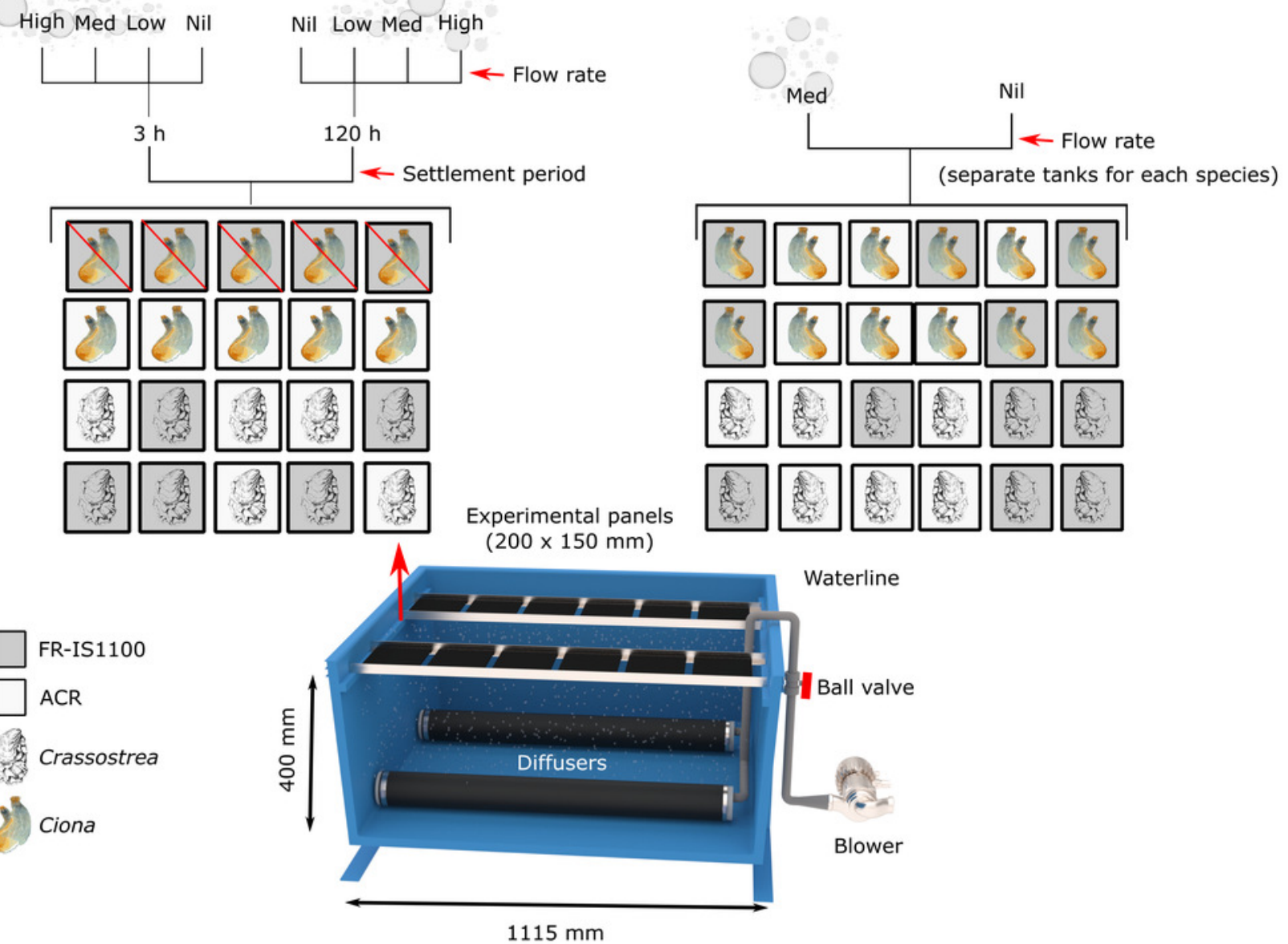




\section{Figure 2}

Schematic of the experimental setup where concrete (CONC) and fouling release (Intersleek 1100SR, FR-IS1100) panels $(300 \times 400 \mathrm{~mm})$ were treated with bubbles while fixed in a vertical orientation.

The same set-up was used for untreated panels (i.e., no bubbles), except frames were deployed without diffusers and the associated pipework.

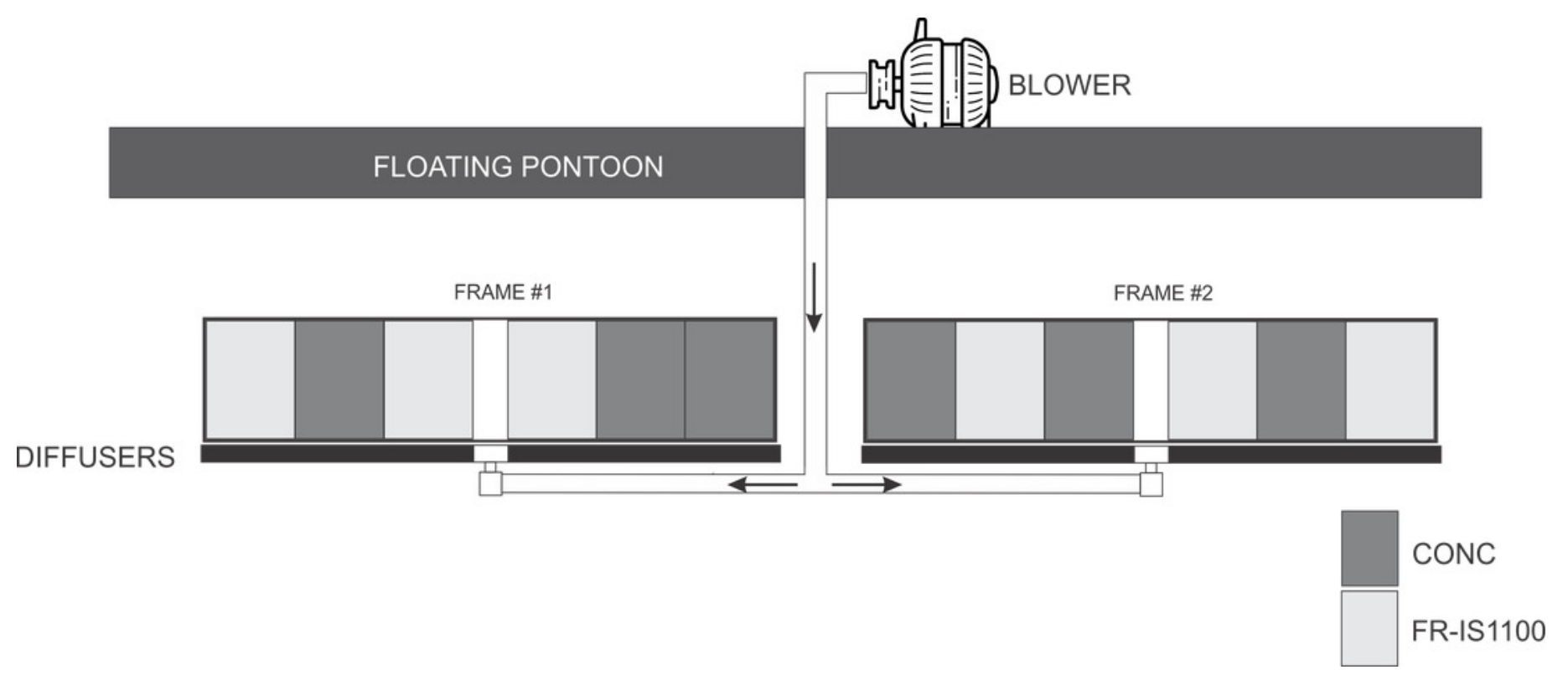


Figure 3

CAD drawing of the experimental raft used in the field trials for horizontal $\left(0^{\circ}\right)$ and angled $\left(22^{\circ}\right)$ surfaces.

Experimental details for the three replicate trials and the final treatment vs control comparison $(n=2)$ are shown. CONC $=$ concrete, $\mathrm{POLY}=$ polyethylene, $\mathrm{FR}-\mathrm{IS} 1000=$ Intersleek 1000, FR-IS1100 = Intersleek 1100SR. 
3 Rounds
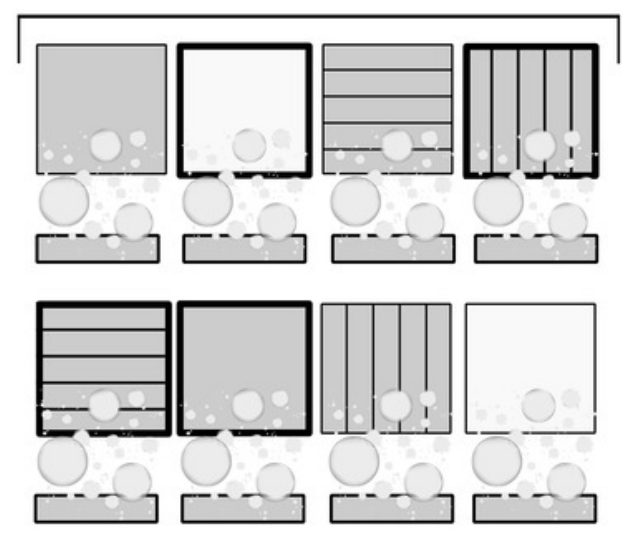

Treatment vs Control

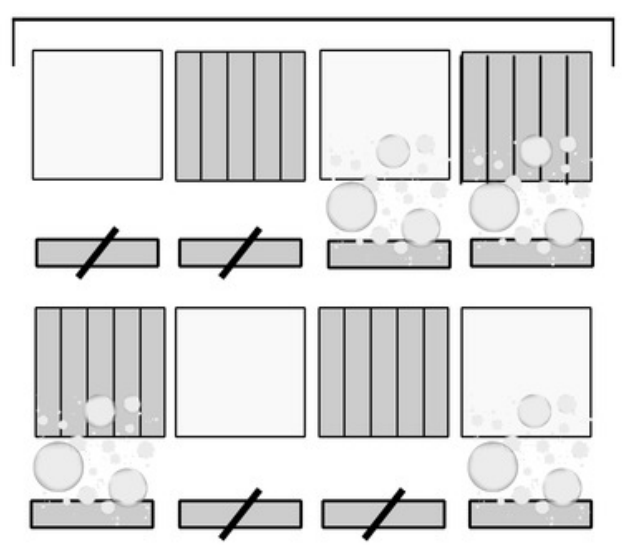

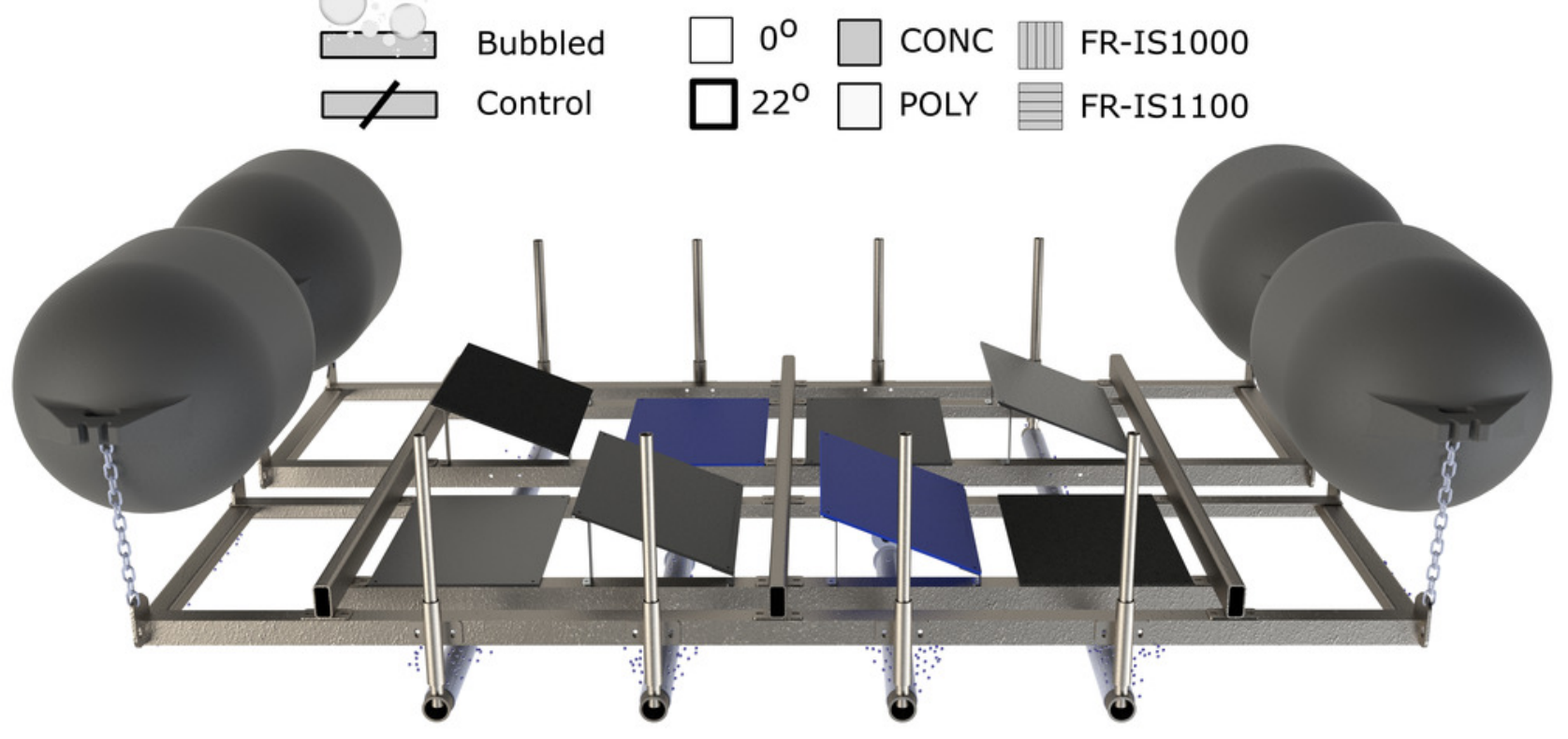




\section{Figure 4}

Number of Ciona remaining on acrylic (ACR) and Crassostrea remaining on acrylic and fouling release (FR-IS1100) panels for both the 3 and $120 \mathrm{~h}$ settlement periods (testing the mechanism of scouring).

Air flow rates at the diffuser: Nil $=$ no bubbles (treatment control), Low $=0.9 \mathrm{~L} \mathrm{~h}^{-1} \mathrm{~cm}^{-2}$, Med

$=1.7 \mathrm{~L} \mathrm{~h}^{-1} \mathrm{~cm}^{-2}$, High $=2.6 \mathrm{~L} \mathrm{~h}^{-1} \mathrm{~cm}^{-2} . \mathrm{N}=5$ per treatment combination. Boxplots display the median, and the first and third quartiles (middle line and lower and upper hinges). The whiskers extend from the hinge to the largest or smallest value no further than $1.5 \times$ the distance between the first and third quartiles. Ciona larval removal was significant for both the 3 and $120 \mathrm{~h}$ settlement periods when exposed to high bubble flow rates, with a reduction of $>90 \%$ of settled larvae relative to the controls ( $p$-value $<0.001$ ). Removal following medium and low flow rates was not significantly lower than the controls, and we did not find an interaction of settlement period and flow rate. For Crassostrea larval removal, we found a significant interaction among flow rate, surface type and settlement period ( $p$-value $<$ 0.001). All flow rates applied to the $120 \mathrm{~h}$ settlement panels were effective at removing larvae from the FR-IS1100 surface. 

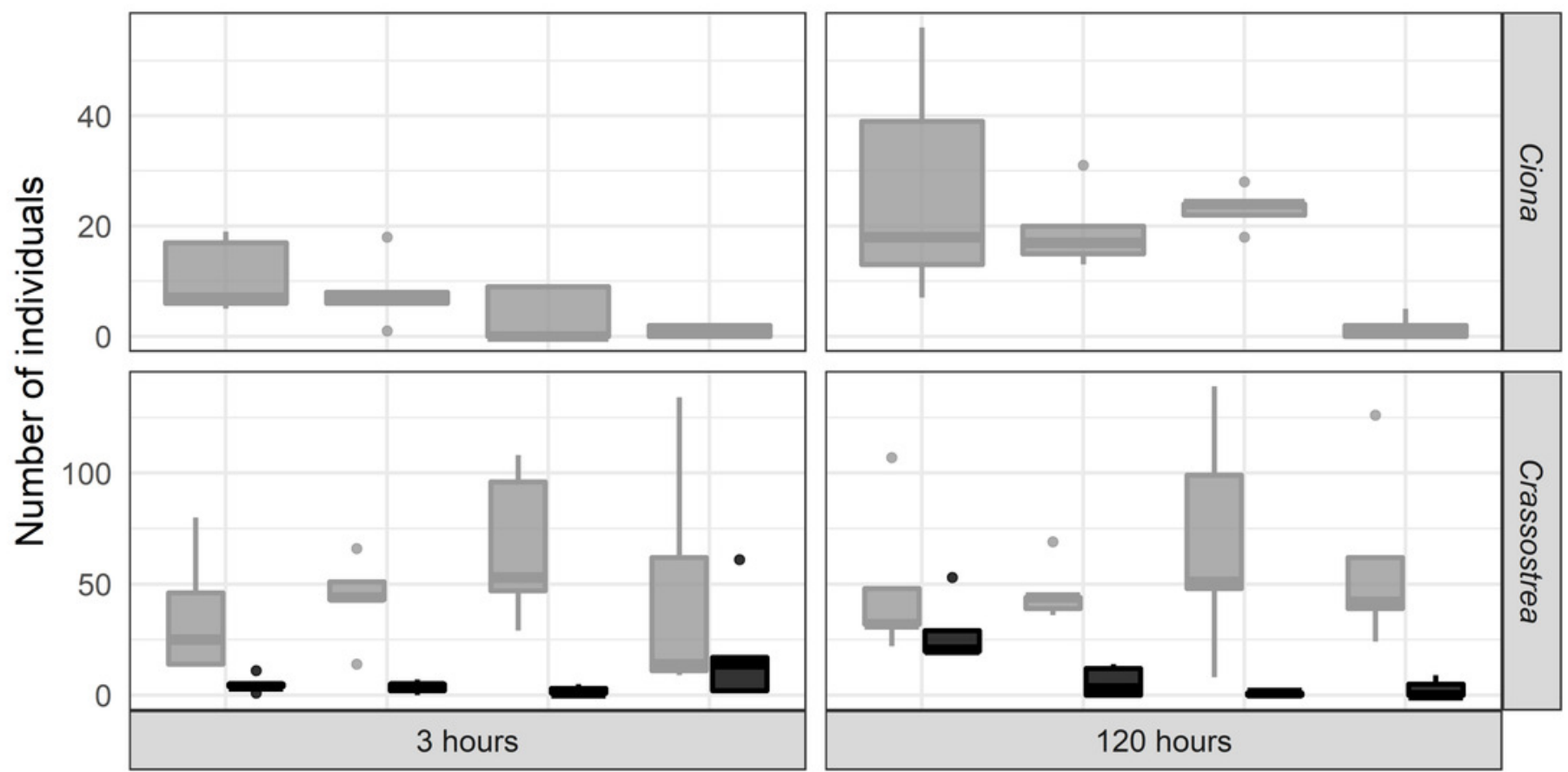

Nil Low Med

High

Flow rate

ACR ש FR-IS1100 


\section{Figure 5}

Number of Ciona and Crassostrea larvae that settled onto blank fouling release (FRIS1100) and acrylic (ACR) panels when exposed to a continuous bubble stream for $24 \mathrm{~h}$ (testing settlement disruption).

$\mathrm{N}=6$ per treatment combination. Ciona and Crassostrea larval densities in the testing system were 50 and $10,500 \mathrm{~L}^{-1}$, respectively. Air flow rates at the diffuser: Nil $=$ no bubbles (treatment control), Med $=1.7 \mathrm{~L} \mathrm{~h}^{-1} \mathrm{~cm}^{-2}$. Boxplots display the median, and the first and third quartiles (middle line and lower and upper hinges). The whiskers extend from the hinge to the largest or smallest value no further than $1.5 \times$ the distance between the first and third quartiles. Panels exposed to continuous bubble streams resulted in nil settlement by Ciona over the $24 \mathrm{~h}$ treatment period. There was also a significant reduction ( $p$-value $<0.001$ ) in Crassostrea settlement compared with the controls. While statistically significant, the treatment effect was not $100 \%$ for Crassostrea. 


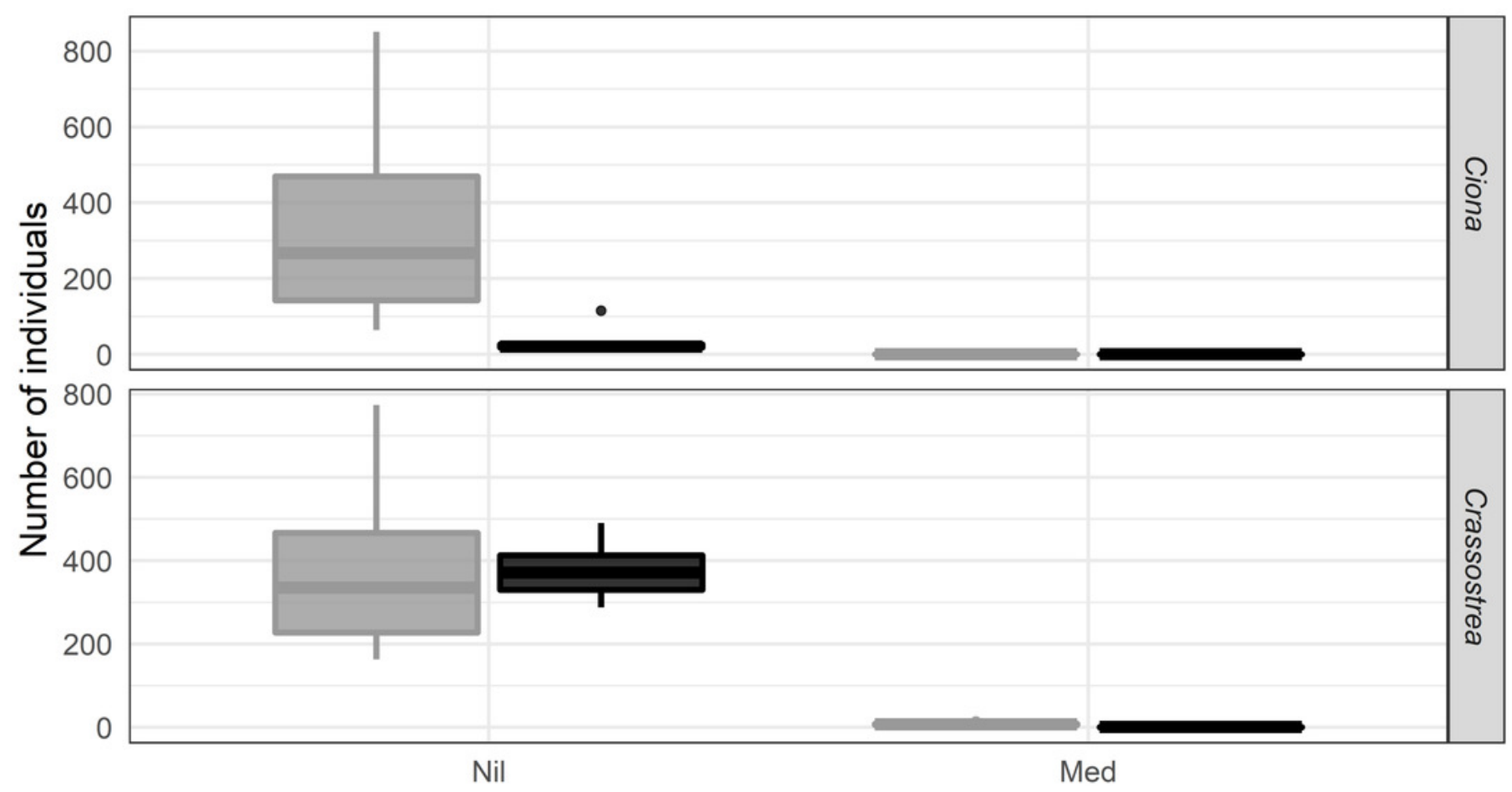

Flow rate

FACR FR-IS1100 


\section{Figure 6}

Effect of bubble streams applied to vertical panels.

Presented as: A) dry weight ( $\mathrm{g}$ ) of biofouling (measured at the end of the experiment), B) percent cover of macrofouling (at end of experiment), and C) quantitative level of fouling (LoF) scores (observations made during the experiment). Total $n=6$ per treatment combination, except treated concrete panels, where $n=3$ due to compromised panels being excluded from analyses. Boxplots display the median dry weight and macrofouling percent cover on untreated (Control) and panels subject to continuous bubble stream treatment (Bubbled) after 13-weeks. The first and third quartiles of the data are shown (middle line and lower and upper hinges). The whiskers extend from the hinge to the largest or smallest value no further than $1.5 \times$ the distance between the first and third quartiles. LoF scores were assigned by divers (weeks 1, 4, 6 and 9 ) and at the surface (week 13). LoF $0=$ no visible fouling (including biofilm), 1 = slime fouling only, 2 = light fouling (1-5\%), 3 = considerable fouling ( $6-15 \%), 4=$ extensive fouling (16-40\%), and 5 = very heavy fouling ( $41-100 \%)$. All models show a significant reduction of fouling on the bubbled treatment in comparison with the control (all p-values $<0.001$ ), and a significant interaction between the bubbled treatment and control and the type of surface ( $p$-value $<0.001$ ). 

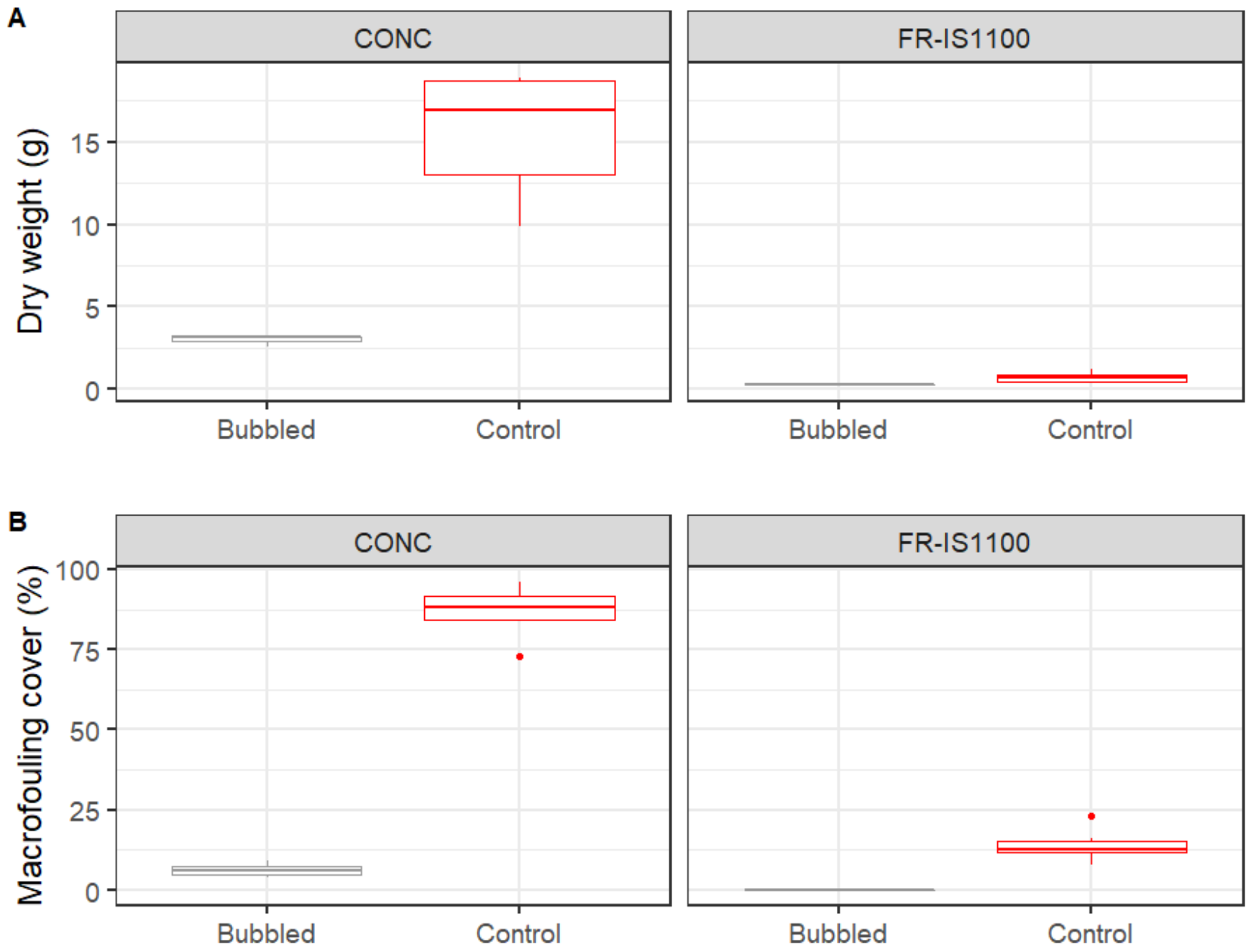

C

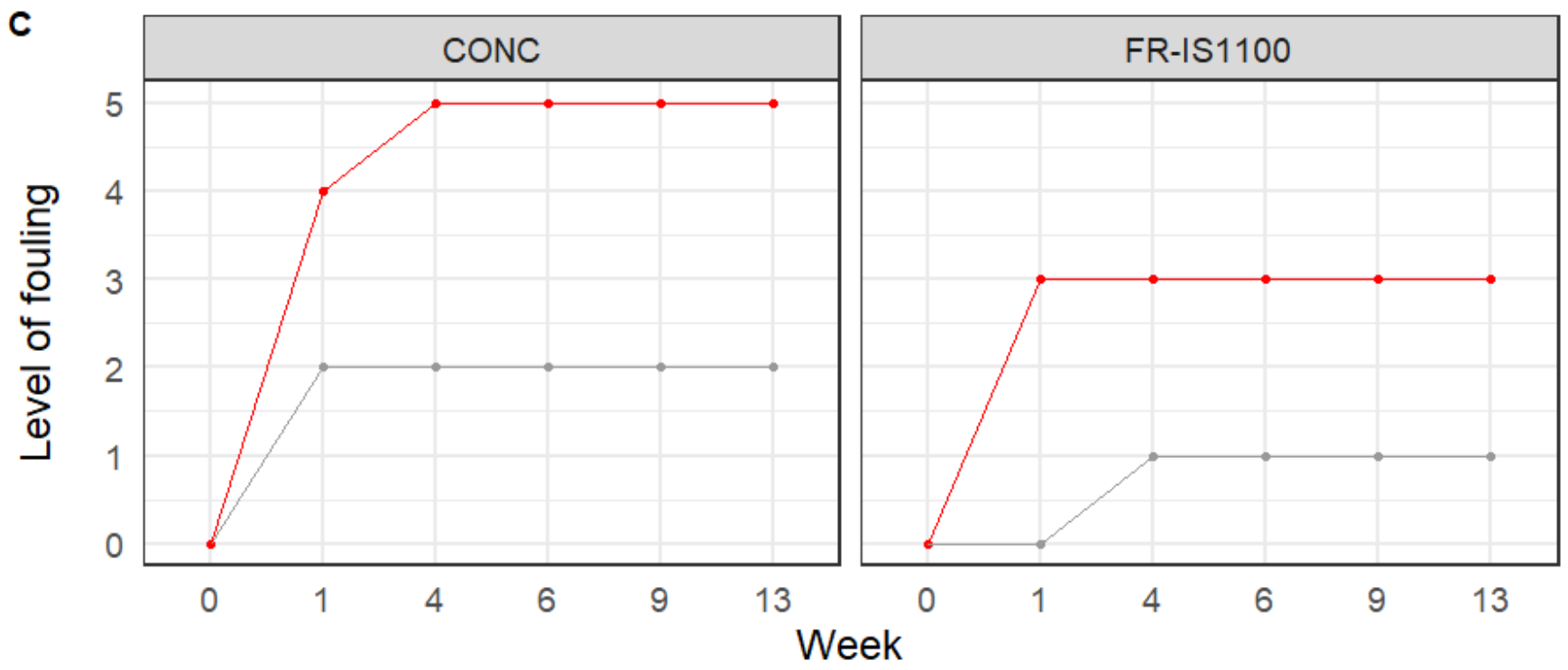

$\because$ Bubbled $\rightarrow$ Control 


\section{Figure 7}

Percent cover of bare space, biofilm and macrofouling on experimental panels subjected to continuous bubble streams.

Four panel types $(\mathrm{CONC}=$ concrete, $\mathrm{POLY}=$ polyethylene, $\mathrm{FR}-\mathrm{IS1000}=$ Intersleek 1000, FRIS1100 $=$ Intersleek 1100 SR) were oriented at two angles $\left(0\right.$ and $\left.22^{\circ}\right)$. The three trials/rounds (R1-3) were undertaken sequentially ( $n=1$ per experimental round). Bubble streams kept macrofouling coverage low, but biofilms typically developed on all surface types. The temporal component was more important in determining the percent cover of bare space, biofilm and macrofouling than the surface type or the position angle, evident by significant differences in percent cover of bare space, biofilm and macrofouling cover between rounds ( $p$-value $<0.001$ ). Biofilm cover differed significantly between some surface types and angles ( $p$-value $<0.001$ ), with highest cover observed on concrete surfaces fixed at $0^{\circ}$. 


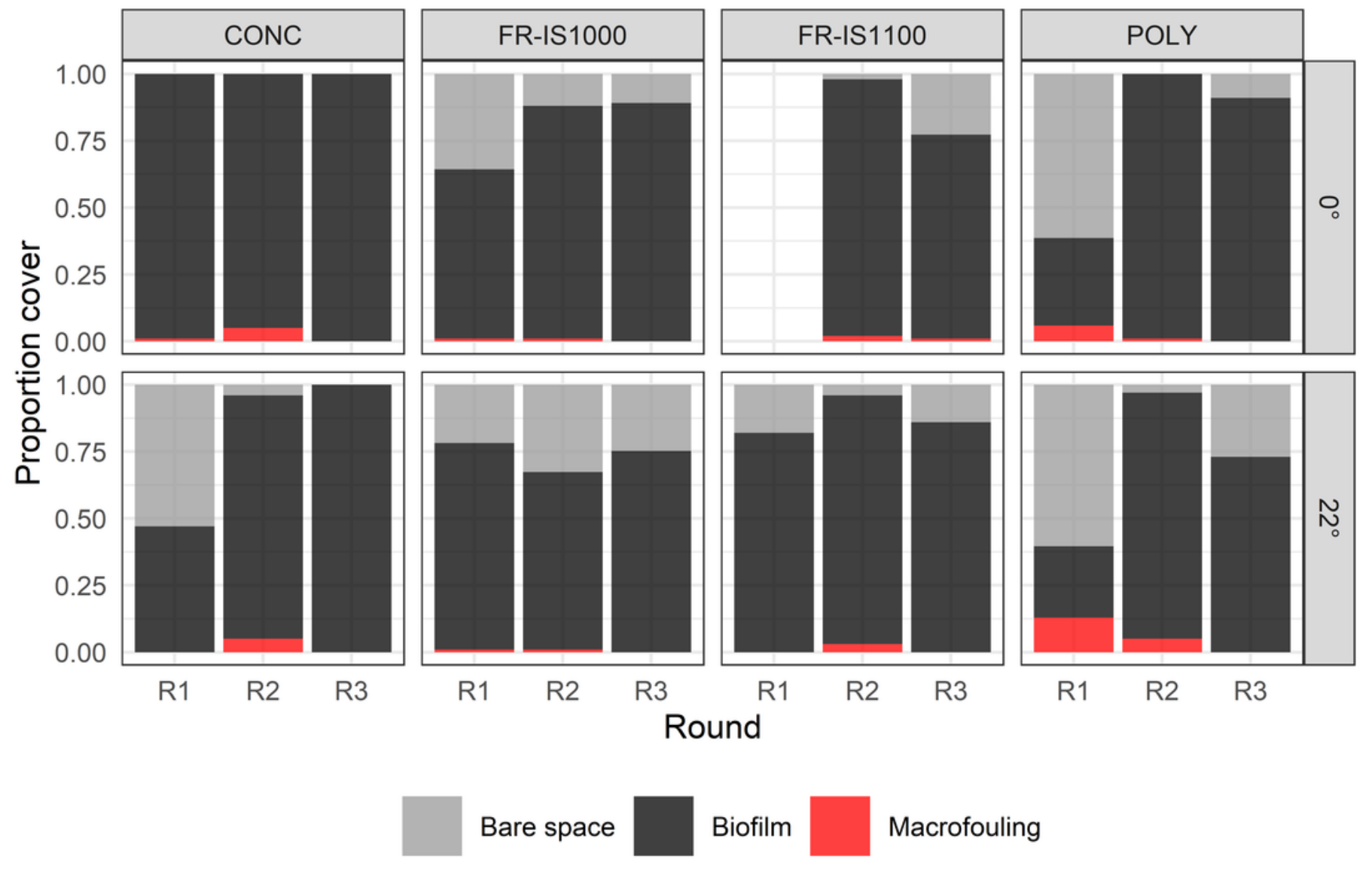




\section{Figure 8}

Percent cover of bare space, biofilm and macrofouling on experimental panels subjected to continuous bubble streams (Bubbled) alongside no treatment (Control).

POLY $=$ polyethylene, FR-IS1000 $=$ Intersleek $1000 . \mathrm{N}=2$ per treatment combination.

Aeration treatment resulted in reduced percent cover of macrofouling and biofilm on the FR-

IS1000 surfaces and reduced macrofouling cover on POLY panels. Macrofouling was not detected on surfaces subjected to treatment, except on areas of the panels where bubble streams were interrupted by fouling on the diffusers or the experimental raft (excluded from analyses). By contrast, the control panels for both surface types were colonized by a range of taxa.
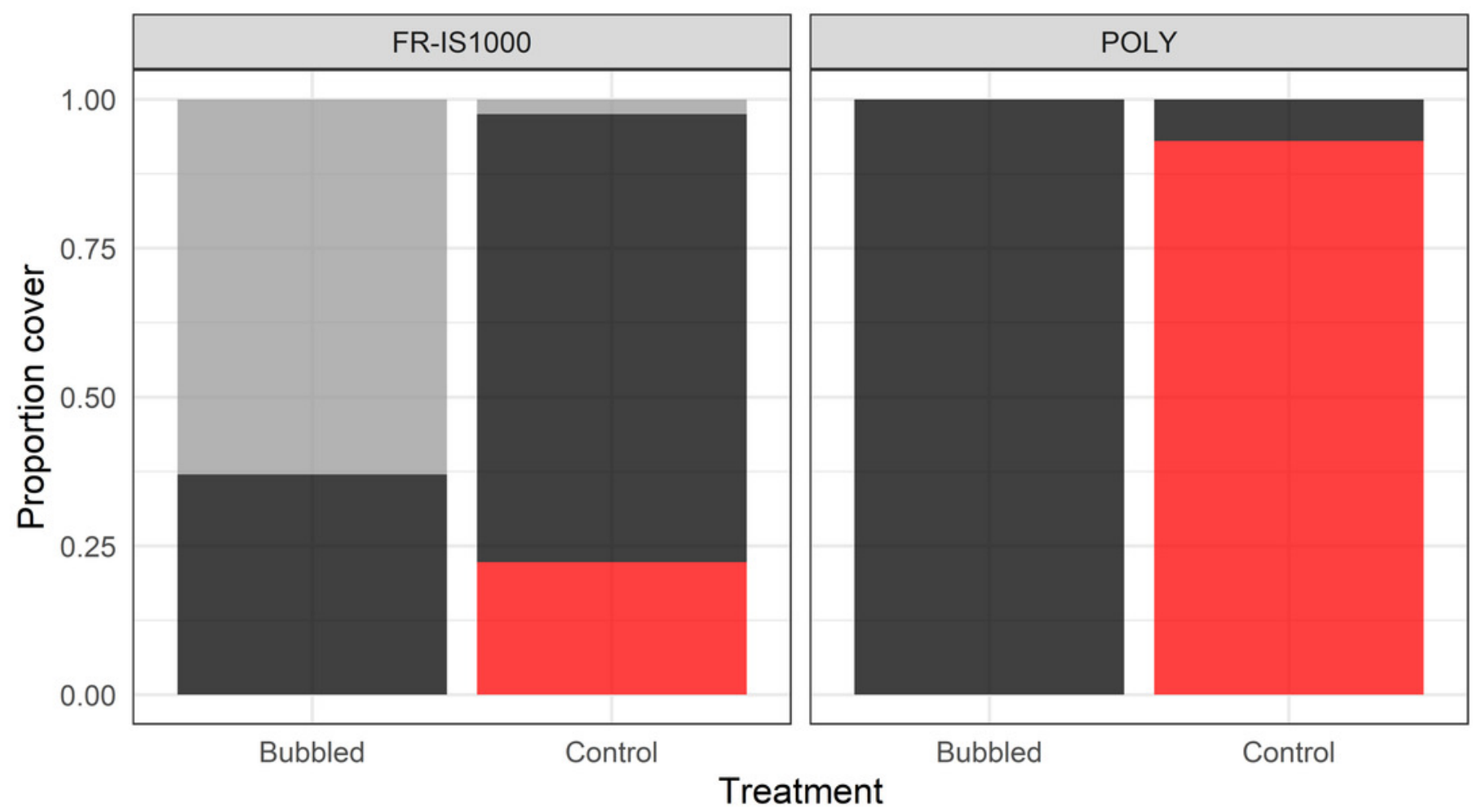

Bare space

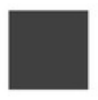

Biofilm

Macrofouling 


\section{Figure 9}

Representative images of biofouling on Intersleek 1000 (FR-IS1000; top row) and polyethylene (POLY; bottom row) panels with (right) and without (left) bubble stream treatment (duration $=119$ days).

The hydroid fouling on the top left corner of the POLY treated panel was caused by fouling on the experimental raft interfering with bubble stream delivery. Similar 'shadows' were evident in the centre of panels immediately above sections of the diffusers that became fouled (see Online Supplementary Material S5). 

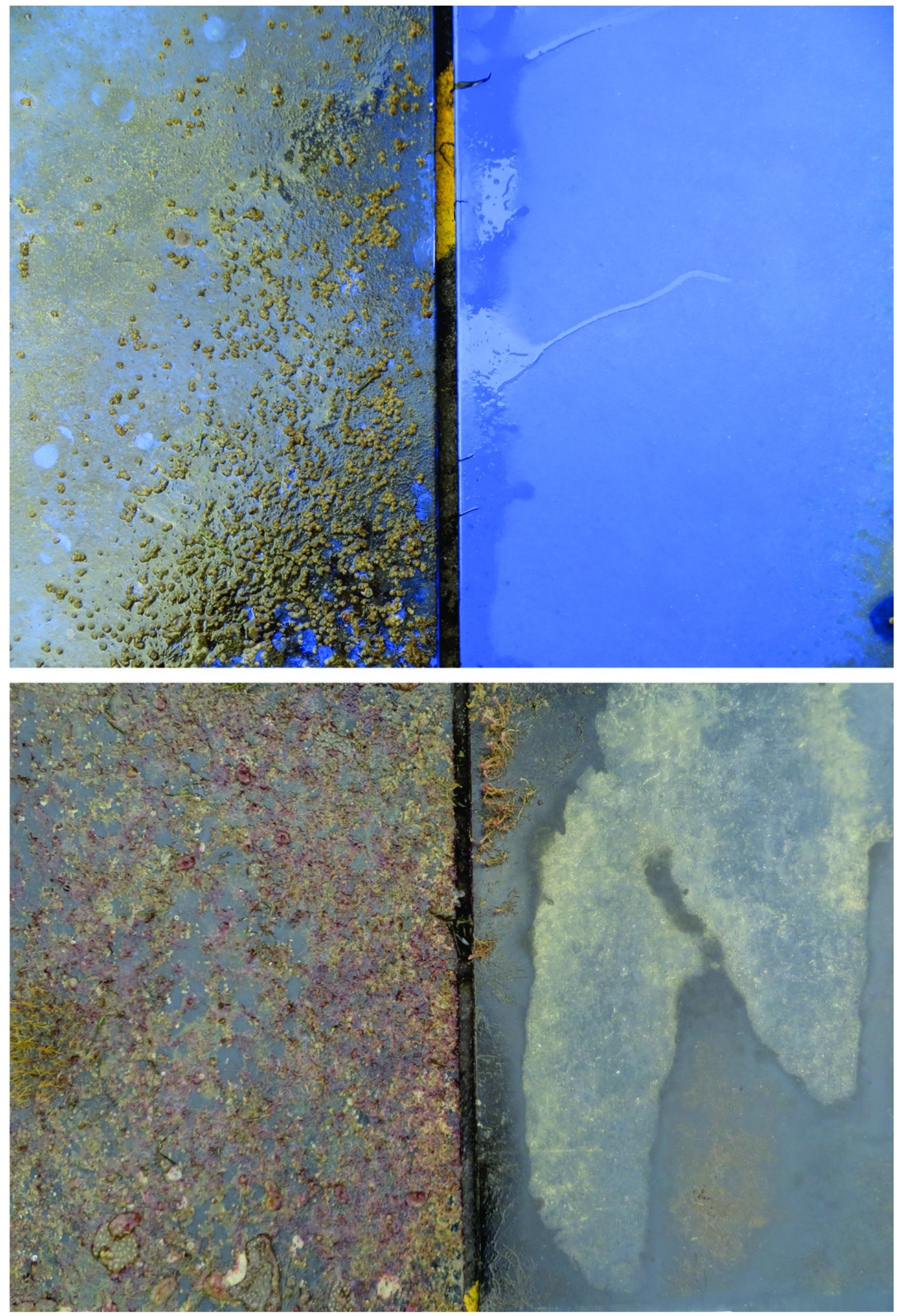


\section{Figure 10}

The small ( $1 \mathrm{~mm}$ ) bubbles from the diffusers were observed joining and forming much larger bubbles on acrylic and fouling release panels $(200 \times 150 \mathrm{~mm}$ ) fixed in a horizontal orientation.

If this could be maintained, it could potentially be exploited to create a barrier between larvae and static coastal infrastructure.

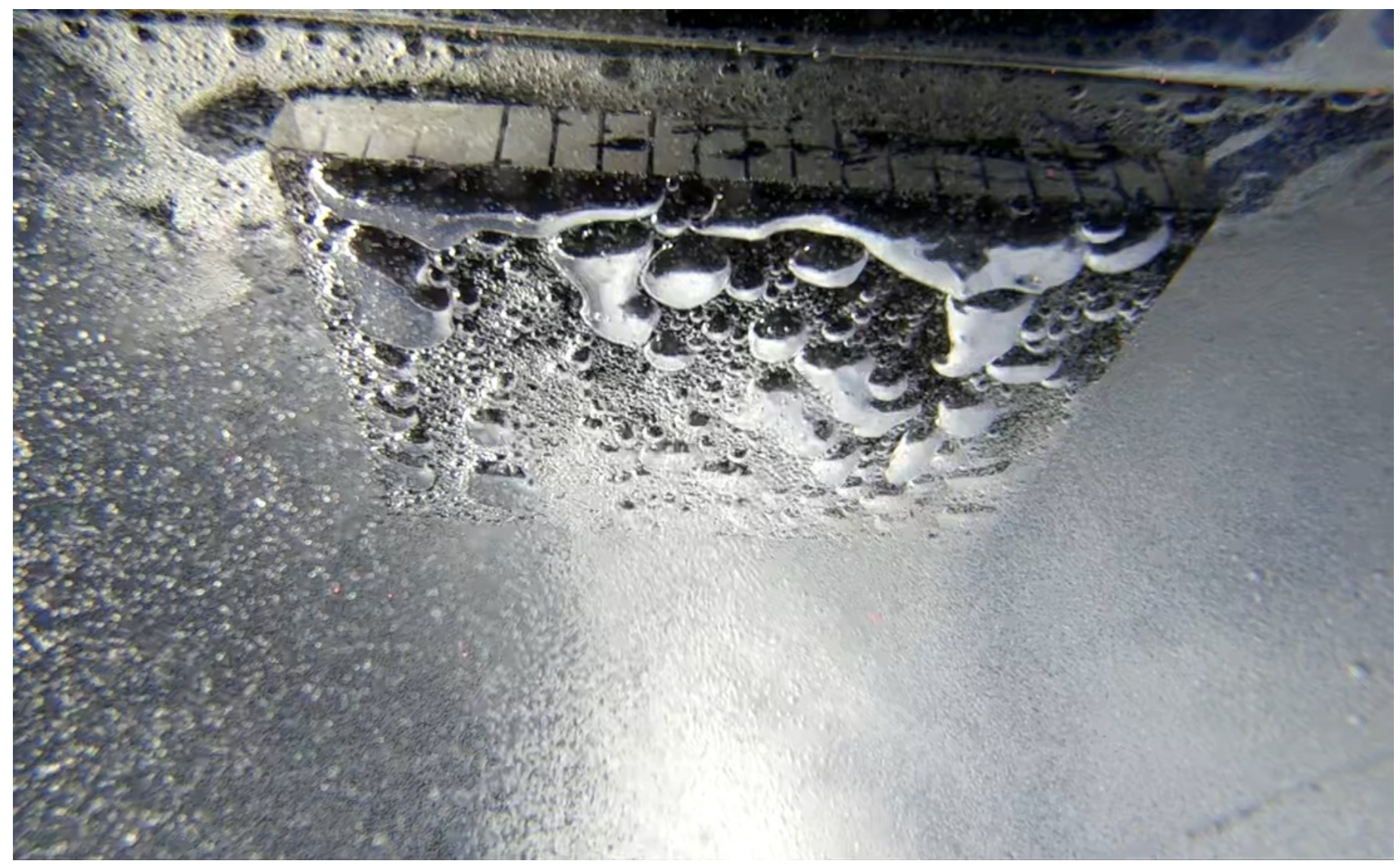




\section{Figure 11}

Extensive biofouling growth developed on the experimental raft (left, dominated by barnacles) and diffusers (right, with extensive hydroid fouling) during each deployment.

Diffuser fouling resulted in sub-optimal delivery of bubbles to overlying panels, evidenced by discrete patches of macrofouling on otherwise clean (i.e., bare space or biofilm only) surfaces. Once formed, macrofouling patches persisted despite ongoing bubble stream treatment.
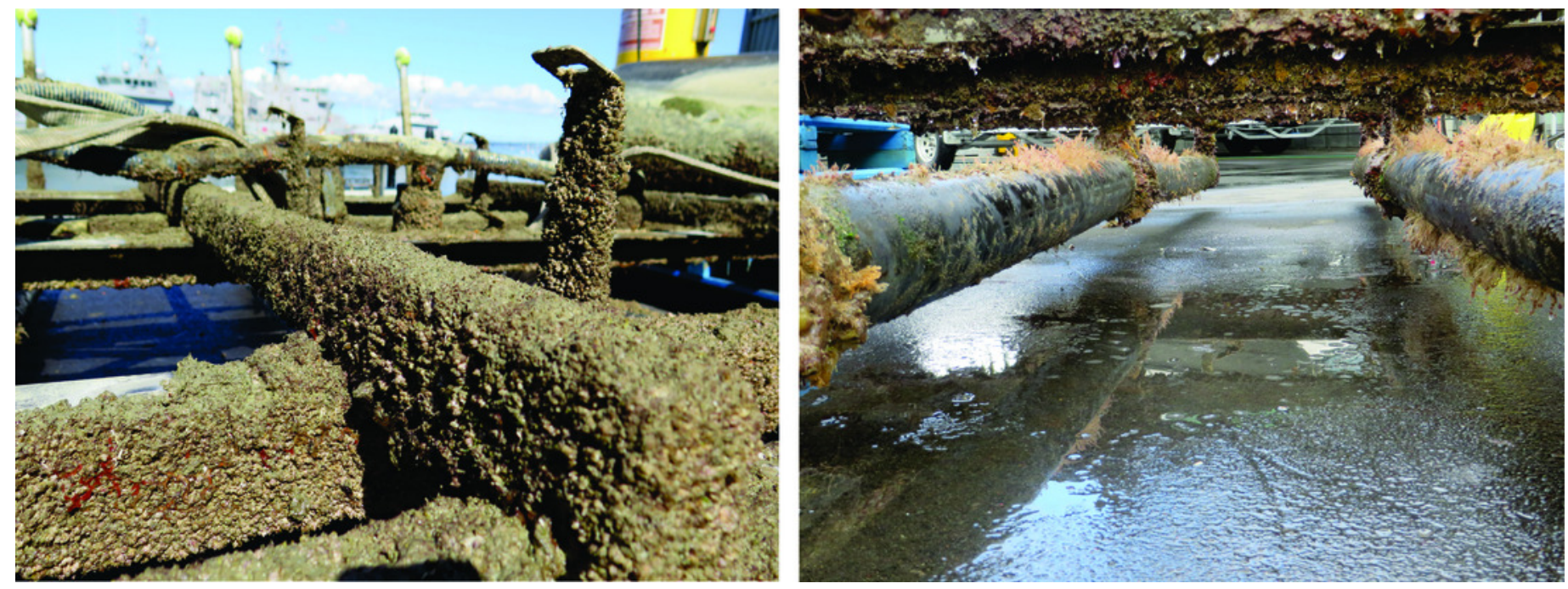